NASA Technical Memorandum 100004

\title{
Comparative Study of High- Resolution Shock-Capturing Schemes for a Real Gas
}

J.-L. Montagné, H. C. Yee, and M. Vinokur

July 1987

(HASA-Ta-100004) CCHFARATIVE STUDY OP BIGH-RESOLOTICE SBOCK-CAPTURIMG SCBEABS FOR

s $87-27470$

L BEAL Gas (RASA) 37 p Avail: ATIS HC 103/BF 101 CSCL 12A

Unclas 0091307 
NASA Technical Memorandum 100004

\section{Comparative Study of High- Resolution Shock-Capturing Schemes for a Real Gas}

J.-L. Montagné and H. C. Yee, Ames Research Center, Moffett Field, California M. Vinokur, Sterling Software, Palo Alto, California

July 1987

\section{N/SA}

National Aeronautics and

Space Administration 


\title{
COMPARATIVE STUDY OF HIGH-RESOLUTION SHOCK-CAPTURING SCHEMES FOR A REAL GAS $\dagger$
}

\author{
J.-L. Montagné $\ddagger$ and H.C. Yee* \\ NASA Ames Research Center, Moffett Field, CA 94035 USA \\ and \\ M. Vinokur ${ }^{\star \star}$ \\ Sterling Software, Palo Alto, CA 94030 USA
}

\section{Summary}

The recently developed second-order explicit shock-capturing methods of the van Leer, Harten, and Yee types, in conjunction with the generalized flux-vector splittings of Vinokur and Montagné, and a generalized Roe's approximate Riemann solver of Vinokur for a real gas are studied. The comparisons are made on different one-dimensional Riemann (shock-tube) problems for equilibrium air with various ranges of Mach numbers, densities, and pressures. Six different Riemann problems are considered. These tests provide a check on the validity of the generalized formulas, since theoretical prediction of their properties appears to be difficult because of the non-analytic form of the state equation. The numerical results in the supersonic and low-hypersonic regimes indicate that these approaches produce good shock-capturing capability and that the shock resolution is only slightly affected by the state equation of equilibrium air. The difference in shock resolution between the various methods varies slightly from one Riemann problem to another, but the overall accuracy is very similar. For the onedimensional case, the relative efficiency in terms of operation-count for the different methods is within 30\%. The main difference between the methods lies in their versatility in being extended to multidimensional problems with efficient implicit solution procedures.

\section{Introduction}

Several newly developed high-resolution shock-capturing methods [1-5] have been shown to be applicable to many multidimensional fluid dynamics problems for a perfect gas. For problems containing moderate to fairly strong shocks, these methods produce highly accurate solutions near discontinuities [6-10]. These numerical methods belong to the class of total variation diminishing (TVD) schemes.

†An abbreviated version will appear in the proceedings of the 7th GAMM Conference on Numerical Methods in Fluid Mechanics, September 9-11, 1987, Louvain-la-Neuve, Belgium.

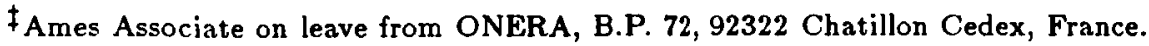

* Research Scientist, Computational Fluid Dynamics Branch

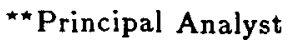


There exists many ways to achieve higher-order spatial accuracy and at the same time have TVD-type properties. Here, two ways are considered. The first way is due to Harten [3], Roe [4] and Yee [5]; the second way is due to van Leer [1] (it is sometimes referred to as the MUSCL approach). Hereinafter, we will refer to the first way as the non-MUSCL approach. To annotate the various algorithms, these nomenclatures will be used. It is emphasized that the basic high-resolution shock-capturing methods for hyperbolic conservation laws are developed for nonlinear scalar hyperbolic conservation laws. Extension of scalar methods to nonlinear systems is accomplished by assuming certain physical models or by local linearization. The mathematical foundation relies mainly on the scalar case. There is no identical theory for nonlinear systems or for the multidimensional counterpart. These schemes are formally extended to one- or higher-dimensional nonlinear systems of hyperbolic conservation laws via the so called Riemann solvers and are evaluated by numerical experiments.

There exist three popular ways of extending scalar schemes to nonlinear systems via the Riemann-solver approaches: the exact Riemann solvers [11], the approximate Riemann solvers [12-15], and the flux-vector splitting techniques [16-18]. However, these Riemann solvers as originally developed, are only valid for a perfect gas. An exact Riemann solver [19], approximate Riemann solver [20-22] and flux-vector splitting approaches [23-25] have recently been generalized to a real gas (see reference [7] for details). The objective of this paper is to investigate the applicability of second-order explicit shock-capturing methods of the van Leer, Harten, Yee types $[1,3-5]$ in conjunction with recently developed generalized flux-vector splittings of Vinokur and Montagné [25] and a generalized Roe's approximate Riemann solver of Vinokur [22] for a real gas.

The combination of the three Riemann solvers and of the differencing algorithms considered above yields five different schemes: a symmetric non-MUSCL scheme [5], an upwind nonMUSCL scheme [7], and three MUSCL-type schemes, depending on the Riemann solvers. The present study provides a check on the validity of the generalized formulas, since theoretical prediction of their properties appears to be difficult because of the non-analytic form of the state equation. The values of the state equation are obtained using a curve-fit procedure given in reference [26]. Comparisons are made on the accuracy and robustness of the methods. The six test cases chosen here are intended to highlight the effect of the high ratios in pressure or density related to shocks, and the effect of departure from perfect gas in the state equation.

A brief description of the Riemann solvers is given in the next section, and the numerical algorithms used in conjunction with the various Riemann solvers are then presented. The test cases and numerical results follow.

\section{Riemann Solvers}

The conservation laws for the one-dimensional Euler equations can be written in the form

$$
\frac{\partial U}{\partial t}+\frac{\partial F(U)}{\partial x}=0
$$

where the column vectors $U$ and $F(U)$ take the form 


$$
U=\left[\begin{array}{c}
\rho \\
m \\
e
\end{array}\right], \quad F=\left[\begin{array}{c}
\rho u \\
m u+p \\
e u+p u
\end{array}\right]
$$

Here $\rho$ is the density, $m=\rho u$ is the momentum per unit volume, $p$ is the pressure, $e=\rho\left(\epsilon+\frac{1}{2} u^{2}\right)$ is the total internal energy per unit volume, and $\epsilon$ is the specific internal energy.

Many Riemann solvers make use of the eigenvalues and eigenvectors of the Jacobian matrix $A=\partial F / \partial U$. For a general gas, one therefore requires the thermodynamic derivatives of $p$. In terms of the internal energy per unit volume $\tilde{\epsilon}=\rho \epsilon$, the thermodynamic derivatives can be defined as

$$
\chi=\left(\frac{\partial p}{\partial \rho}\right)_{\tilde{\epsilon}} ; \quad \kappa=\left(\frac{\partial p}{\partial \widetilde{\epsilon}}\right)_{\rho} .
$$

If $h=\epsilon+p / \rho$ is the specific enthalpy, one can obtain for the speed of sound $c$ the relation

$$
c^{2}=\chi+\kappa h
$$

For a perfect gas, $\chi=0$, and $\kappa=(\gamma-1)$.

The Jacobian matrix $A$ takes the form

$$
A=\left[\begin{array}{ccc}
0 & 1 & 0 \\
\chi-(2-\kappa) u^{2} / 2 & (2-\kappa) u & \kappa \\
\left(\chi+\kappa u^{2} / 2-H\right) u & H-\kappa u^{2} & (1+\kappa) u
\end{array}\right]
$$

where $H=h+u^{2} / 2$ is the total enthalpy. The three eigenvalues of $A$ are

$$
a^{1}=u-c, \quad a^{2}=u, \quad \text { and } a^{3}=u+c .
$$

The corresponding right-eigenvector matrix is

$$
R=\left[\begin{array}{ccc}
1 & 1 & 1 \\
u-c & u & u+c \\
H-u c & \frac{u^{2}}{2}-\frac{\chi}{\kappa} & H+u c
\end{array}\right]
$$

and its inverse can be written as

$$
R^{-1}=\left[\begin{array}{ccc}
\frac{1}{2}\left(b_{1}+\frac{u}{c}\right) & -\frac{1}{2}\left(u b_{2}+\frac{1}{c}\right) & \frac{b_{2}}{2} \\
1-b_{1} & b_{2} u & -b_{2} \\
\frac{1}{2}\left(b_{1}-\frac{u}{c}\right) & -\frac{1}{2}\left(u b_{2}-\frac{1}{c}\right) & \frac{b_{2}}{2}
\end{array}\right],
$$

where $b_{1}=\frac{1}{c^{2}}\left[\kappa u^{2} / 2+\chi\right]$ and $b_{2}=\kappa / c^{2}$. In order to relate the variables $p$ and $c$ to the independent variables $\rho$ and $\epsilon$, it is customary to introduce the nondimensional thermodynamic variables

$$
\bar{\gamma}=1+\frac{p}{\rho \epsilon}, \quad \gamma=\frac{\rho c^{2}}{p}
$$


Since there is an arbitrary additive constant in the definition of $\epsilon, \bar{\gamma}$ does not have a well defined value. In practice, it is introduced only when the arbitrary constant has been chosen so that $\epsilon \rightarrow 0$ when $p \rightarrow 0$. The expressions for flux-vector splittings derived below will be given in term of a general $\epsilon$. Only in the numerical results will $\bar{\gamma}$ be employed. For a perfect gas the variables $\gamma$ and $\bar{\gamma}$ are constant and equal to each other. For an equilibrium real gas they are both arbitrary functions of $\rho$ and $\epsilon$.

\subsection{An Approximate Riemann Solver (Generalized Roe Average [22])}

Among the various approximate Riemann solvers for a perfect gas [12-15], the most common one uses the Roe average [12] because of its simplicity and its ability to satisfy the jump conditions. The generalization of the Roe average for a real gas has been carried out by Vinokur [22] for the three-dimensional case. A summary of the generalized Roe-average derivation for the one-dimensional case is given below. The use of the approximate Riemann solver in conjunction with the numerical schemes will be discussed in sections 4.1 and 4.2 .

The flux at a point separating two states $U^{L}$ and $U^{R}$ is based on the eigenvalues and eigenvectors of some average $\bar{A}$. The optimum choice for $\bar{A}$ is one satisfying

$$
\Delta F=\vec{A} \Delta U
$$

where $\Delta(\cdot)=(\cdot)^{R}-(\cdot)^{L}$. This choice of $\bar{A}$ will capture discontinuities exactly. One way of obtaining $\bar{A}$ is to seek an average state $\bar{U}$, such that

$$
\overline{\boldsymbol{A}}=\boldsymbol{A}(\bar{U}) .
$$

Such a state is known as a Roe-averaged state. Expressions for a perfect gas were first devised by Roe $[12]$.

The entries in $A$ depend explicitly on the thermodynamic variables $h, \chi$, and $\kappa$. Since the density is not explicitly required, one would expect the Roe-averaged state to depend on $\rho^{L}$ and $\rho^{R}$ through their ratio only. It is, therefore, convenient to define the parameter $D=\sqrt{\rho^{R} / \rho^{L}}$. We first examine the second component of equation (3.9). The average velocity $\bar{u}$ must be a linear combination of $u^{L}$ and $u^{R}$. Recalling that $u^{L}$ and $u^{R}$ can be independently prescribed, we can readily establish the same $\bar{u}$ as for a perfect gas:

$$
\bar{u}=\frac{u^{L}+D u^{R}}{1+D} .
$$

This definition will satisfy all the terms involving the velocity. Note that $\bar{u}$ always lies between $u^{L}$ and $u^{R}$. The remaining terms in the equation result in the condition

$$
\bar{\chi} \Delta \rho+\bar{\kappa} \Delta \tilde{\epsilon}=\Delta p .
$$

This last condition is automatically satisfied for a perfect gas.

In order to satisfy the third component of equation (3.9) we also require $\bar{H}$ to have the same form as the perfect-gas version:

$$
\bar{H}=\frac{H^{L}+D H^{R}}{1+D}
$$


Using the definition of $H$, equations (3.11) and (3.13) can be combined to define the Roeaveraged specific enthalpy as

$$
\bar{h}=\frac{h^{L}+D h^{R}}{1+D}+\frac{D}{2(1+D)^{2}}(\Delta u)^{2} .
$$

Note that $\bar{h}$ could lie outside the range given by $h^{L}$ and $h^{R}$ if $\Delta u$ is sufficiently large. The Roe-averaged sound speed is given by equation (3.3) as

$$
\bar{c}^{2}=\bar{\chi}+\bar{\kappa} \bar{h} .
$$

For a perfect gas, equations (3.11) through (3.15) are sufficient to define uniquely $\bar{a}^{l}, \bar{R}$, and $\bar{R}^{-1}$, since $\bar{\chi}=0$ and $\bar{\kappa}$ is a given constant.

For a real gas, equation (3.12) provides only one relation for the variables $\bar{\chi}$ and $\bar{\kappa}$. Since the equation of state can be nonconvex, a universally valid unique solution may not be possible. In order to gain some insight, we consider the special case in which the states $R$ and $L$ are precisely those that satisfy the jump conditions across a discontinuity (either a contact discontinuity or a shock wave). Then equations (3.9) through (3.15) are consistent with the exact Riemann solver even though $\bar{\chi}$ and $\bar{\kappa}$ are not uniquely defined. For a shock wave one obtains

$$
\bar{h}=\frac{h^{L}+D^{2} h^{R}}{1+D^{2}}
$$

and

$$
\bar{c}^{2}=\frac{\Delta p}{\Delta \rho} .
$$

For the special case of a thermally perfect gas, $c^{2}$ is a function of $h$ only, and one can readily show that the values of $\bar{h}$ and $\bar{c}^{2}$ given by equations (3.16) can only satisfy this law if the function is linear. But this is precisely the definition of a perfect gas.

The above analysis makes it clear that for a real gas the values of $\bar{\chi}$ and $\bar{\kappa}$ must be defined in terms of the thermodynamic states $R$ and $L$, and not in terms of the state $\bar{h}$. Because of the nonconvex nature of a real-gas equation of state, the values of $\chi$ and $\kappa$ at states $R$ and $L$, or some average of the two, will not satisfy equation (3.12), in general. One way to obtain unique values of $\bar{\chi}$ and $\bar{\kappa}$ is to project the average state given by $R$ and $L$ onto the straight line defined by equation (3.12). Since the value of $\Delta \tilde{\epsilon}$ depends on the arbitrary constant in the definition of $\epsilon$, the resulting value of $\bar{\kappa}$ will depend on the choice of this constant. To overcome this arbitrariness, one divides equation (3.12) by $\kappa$. The straight line for the variables $1 / \kappa$ and $\chi / \kappa$ has a slope given by $\Delta p$ and $\Delta \rho$, both of which are uniquely defined by states $R$ and $L$. Since $\Delta p$ and $\Delta \rho$ are not dimensionally consistent, one must further introduce a scale factor proportional to their ratio in order to have the final answer independent of the choice of dimensional units. A natural candidate is the square of an average sound speed. In terms of arithmetic averages, and the scale factor $\widehat{c}^{2}=\left[\left(c^{L}\right)^{2}+\left(c^{R}\right)^{2}\right] / 2$, one obtains the expressions

$$
\frac{1}{\bar{\kappa}}=\left\{\frac{1}{2}\left[\left(\frac{\chi^{L}}{\kappa^{L}}+\frac{\chi^{R}}{\kappa^{R}}\right) \Delta p+\left(\frac{1}{\kappa^{L}}+\frac{1}{\kappa^{R}}\right) \tilde{c}^{4} \Delta \rho\right] \Delta \rho+\Delta \tilde{\epsilon} \Delta p\right\} /\left(\Delta p^{2}+\widehat{c}^{4} \Delta \rho^{2}\right)
$$


and

$$
\frac{\bar{\chi}}{\bar{\kappa}}=\left\{\frac{1}{2}\left[\left(\frac{\chi^{L}}{\kappa^{L}}+\frac{\chi^{R}}{\kappa^{R}}\right) \Delta p+\left(\frac{1}{\kappa^{L}}+\frac{1}{\kappa^{R}}\right) \widehat{c}^{4} \Delta \rho\right] \Delta p-\widehat{c}^{4} \Delta \tilde{\epsilon} \Delta \rho\right\} /\left(\Delta p^{2}+\widehat{c}^{4} \Delta \rho^{2}\right) .
$$

Other expressions can be obtained by using different scalings and taking different types of averages. The optimum choice that would be valid for a wide range of conditions will require further research and numerical experiments.

\subsection{Generalized Steger and Warming Flux-Vector Splitting [25]}

For subsonic flow, the eigenvalues of $A$ are of mixed sign. In flux-vector splitting methods, the flux $F$ is divided into several parts, each of which has a Jacobian matrix whose eigenvalues are all of one sign. The flux-vector splitting approach of Steger and Warming [16] made use of the assumption that the Euler equations are homogeneous of degree one. This homogeneity property is satisfied for a thermally perfect gas. While they only gave the results for a perfect gas, the results for a thermally perfect gas are found in reference [25]. The generalization to an arbitrary equilibrium real gas presented here makes direct use of the eigenvalues of $A$, and is an extension of the work of Sanders and Prendergast [18] and Montagné [22]. The basic idea is the observation that the eigenvalues are actually three velocities. We can associate with each eigenvalue $a^{l}, l=1,2,3$, a fictitious stream with velocity $a^{l}$, and some unknown density $\rho^{l}$ and specific internal energy $\epsilon^{l}$. Each stream can then be characterized by a column vector

$$
U^{l}=\left[\begin{array}{c}
\rho^{l} \\
m^{l} \\
e^{l}
\end{array}\right]
$$

where $m^{l}=\rho^{l} a^{l}$ and $\left.e^{l}=\rho^{l} \mid \epsilon^{l}+\frac{1}{2}\left(a^{l}\right)^{2}\right\}$. The fictitious flux associated with each stream is assumed to be convective only, namely,

$$
F^{l}=a^{l} U^{l}
$$

The six unknowns are determined from the conditions

$$
U=\sum_{l=1}^{3} U^{l} \quad \text { and } \quad F=\sum_{l=1}^{3} F^{l} .
$$

Since the second component of $U$ and the first component of $F$ give the same equation, we are left with one degree of freedom. From the first two components of $U$ and $F$, one readily obtains

$$
\rho^{1}=\rho^{3}=\frac{\rho}{2 \gamma} \quad \text { and } \quad \rho^{2}=\rho\left(1-\frac{1}{\gamma}\right) .
$$

The third components of $U$ and $F$ result in the relations

$$
\epsilon^{1}=\epsilon^{3} \quad \text { and } \quad \sum_{l=1}^{3} \rho^{l} \epsilon^{l}=\rho\left(\epsilon-\frac{c^{2}}{2 \gamma}\right) .
$$


The relations in equations (3.21a) and (3.21b) have the same form as those for the StegerWarming solution for a thermally perfect gas. In choosing the manner in which the remaining degree of freedom is expressed, we are guided by the fact that $\epsilon$ is not an absolute quantity. Consequently, we express the $\epsilon^{i}$ as

$$
\epsilon^{2}=\epsilon-\frac{(1-\alpha) c^{2}}{\gamma(\gamma-1)}, \quad \text { and } \quad \epsilon^{1}=\epsilon^{3}=\epsilon+\left(\frac{1-\alpha}{\gamma}-\frac{1}{2}\right) c^{2} .
$$

Here the non-dimensional thermodynamic parameter $\alpha$ has been chosen so that $\alpha=0$ corresponds to the Steger-Warming solution for a thermally perfect gas. The final expressions for the $F^{l}$ can be written in the form

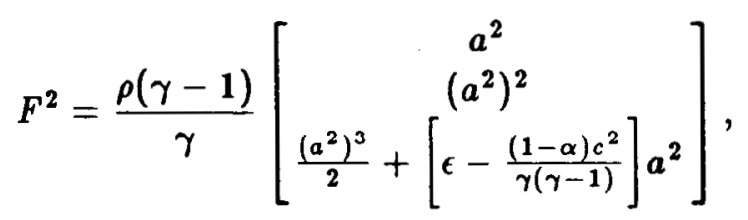

and

$$
F^{l}=\frac{\rho}{2 \gamma}\left[\begin{array}{c}
a^{l} \\
\left(a^{l}\right)^{2} \\
\frac{\left(a^{l}\right)^{3}}{2}+\left[\epsilon+\left(\frac{1-\alpha}{\gamma}-\frac{1}{2}\right) c^{2}\right] a^{l}
\end{array}\right]
$$

for $l=1$ or 3 . We thus have a one-parameter family of flux-vector splittings, where $\alpha$ can be an arbitrary parameter. The total flux can be split according to the sign of the eigenvalues. For $-c<u<0$ we, therefore, have

$$
F^{+}=F^{3} \quad \text { and } \quad F^{-}=F^{1}+F^{2}
$$

and for $0<u<c$

$$
F^{+}=F^{2}+F^{3} \quad \text { and } \quad F^{-}=F^{1}
$$

For a perfect gas one can show that when $-c<u<0$, the determinant of the split-flux Jacobian $A^{+}=\partial F^{+} / \partial U$ is

$$
\operatorname{det}\left(A^{+}\right)=\frac{(c+u)^{3}}{16 \gamma^{2}}\left[3-\gamma-2 \alpha \frac{(\gamma-1)}{\gamma}\right] .
$$

The determinant is the product of the three eigenvalues, and a necessary condition for $\mathrm{A}^{+}$to have eigenvalues that are all positive is that $\operatorname{det}\left(A^{+}\right)>0$. It follows from equation (3.24a) that we must take $\alpha<\gamma(3-\gamma) /[2(\gamma-1)]$. For the region $0<u<c$, one can show that the minimum of $\operatorname{det}\left(A^{+}\right)$occurs when $u$ approaches zero. This minimum value is

$$
\operatorname{det}\left(A^{+}\right)=\frac{c^{3}}{16 \gamma^{3}}\left[5-3 \gamma-2 \alpha \frac{(3-\gamma)(\gamma-1)}{\gamma}\right] .
$$


The condition $\operatorname{det}\left(A^{+}\right)>0$ requires that $\alpha<\gamma(5-3 \gamma) /[2(3-\gamma)(\gamma-1)]$. For $1<\gamma<3$, this second condition on $\alpha$ automatically satisfies the previous inequality derived for $-c<u<0$. In order to determine an optimum value of $\alpha$, we note that in general the three eigenvalues of $A^{+}$are discontinuous at $-c, 0$, and $c$. For $\alpha=0$, two of the eigenvalues are continuous at $u=c$, and one can further show that all the eigenvalues are real and non-negative in the subsonic region when $\gamma<5 / 3$. For a thermally perfect gas with vibrational excitation, one can show that all the eigenvalues remain real and non-negative when $\alpha=0$. Because of the general nature of the variation of $\gamma$ and $\epsilon$, it is simplest to set $\alpha=0$ for an arbitrary equilibrium real gas. The numerical results presented below were actually based on an earlier parameterization which was chosen to reduce to the perfect-gas solution. It corresponds to the present $\alpha=(\bar{\gamma}-\gamma) /(\bar{\gamma}-1)$. The cases were also rerun with $\alpha=0$, and no noticeable difference was observed.

\subsection{Generalized van Leer Flux-Vector Splitting [25]}

In a different approach, van Leer [17] constructed a flux-vector splitting for a perfect gas in terms of low-order polynomials of $u$, which gives continuous eigenvalues at $u=0$ and $u= \pm c$. The splitting also has the desirable property that one of the eigenvalues of the split -flux Jacobians is identically zero. This results in a sharper capture of transonic shocks. An extension of van Leer's splitting for a real gas was derived by Montagné [24], but it is not internally consistent. The present formulas represent the most general, consistent extension of van Leer's splitting for a real gas. Because of the arbitrary nature of a real-gas law, the condition of one eigenvalue being identically zero cannot be satisfied exactly.

For $|u|<c$, the continuity requirements necessitate a factor $(u \pm c)^{2}$ in the formulas for $F^{ \pm}$. The expressions for the first two components of $F^{ \pm}$that are given by the lowest-order polynomials in $u$ are readily found to be

$$
F_{m a s s}^{ \pm}= \pm \frac{\rho}{4 c}(u \pm c)^{2}
$$

and

$$
F_{m o m}^{ \pm}=\frac{\rho}{\gamma 4 c}(u \pm c)^{2}[2 c \pm(\gamma-1) u]
$$

For a perfect gas they are identical to those derived by van Leer. The expression for the third component, which satisfies continuity and symmetry conditions, can be written most generally in the form

$$
\begin{aligned}
F_{\text {ene }}^{ \pm}= & \pm \frac{1}{2\left(\gamma^{2}-1\right)} \frac{\rho}{4 c}(u \pm c)^{2}[2 c \pm(\gamma-1) u]^{2} \pm \frac{\rho}{4 c}(u \pm c)^{2}\left[\epsilon-\frac{c^{2}}{\gamma(\gamma-1)}\right] \\
& \pm \beta \frac{\rho}{4 c}(u \pm c)^{2}(u \mp c)^{2},
\end{aligned}
$$

where the arbitrary parameter $\beta$ has been chosen so that $\beta=0$ corresponds to van Leer's solution for a perfect gas. We again have a one-parameter family of flux-vector splittings.

For a perfect gas, the second term in $F_{\text {ene }}^{ \pm}$vanishes. Van Leer's condition of a vanishing eigenvalue for $A^{ \pm}$requires $\beta$ to vanish also, so that $F_{\text {ene }}^{ \pm}$reduces to one term. One can readily 
show that the remaining eigenvalues are both of the proper sign for $1 \leq \gamma \leq 3$. For a real gas, one requires at least two terms for $F_{\text {ene. }}^{ \pm}$. (The one-term solution of Montagné [24] actually violates the condition $F=F^{+}+F^{-}$). Because of the general nature of the variations of $\gamma$ and $\epsilon$, it is impossible to obtain the vanishing eigenvalue condition identically throughout the velocity range, for any choice of $\beta$.

One can demonstrate readily that for a thermally perfect gas, the two-term solution has one eigenvalue that is of the wrong sign for the whole subsonic velocity range. Fortunately, the magnitude of the offending eigenvalue is extremely small, so that its effect on a numerical scheme is not noticeable. In view of this fact, and because of the general nature of the variation of $\gamma$ and $\epsilon$, it is simplest to set $\beta=0$ for a real gas. The results presented in section $V$ are obtained with this value of $\beta$ and seem to show that this approximation is valid for a wide range of flow conditions.

\section{Description of the Numerical Algorithms}

Let $U_{j}^{n}$ be the numerical solution at $x=j \Delta x, t=n \Delta t$, with $\Delta x$ the spatial mesh size and $\Delta t$ the time-step. The second-order in time and second- or third-order in space, explicit difference schemes considered here for both the MUSCL and the non-MUSCL approaches can be written in the following form

$$
U_{j}^{n+1}=U_{j}^{n}-\lambda\left(\tilde{H}_{j+\frac{1}{2}}^{n}-\tilde{H}_{j-\frac{1}{2}}^{n}\right)
$$

where $\lambda=\Delta t / \Delta x$. The vector functions $\tilde{H}_{j \pm \frac{1}{2}}$ are sometimes referred to as numerical flux functions.

\subsection{Non-MUSCL Approach}

The numerical flux function $\tilde{H}_{j \pm \frac{1}{2}}$ for a non-MUSCL-type approach for both the upwind and symmetric TVD schemes [6] using an approximate Riemann solver can be expressed as

$$
\tilde{H}_{j+\frac{1}{2}}=\frac{1}{2}\left[F\left(U_{j}\right)+F\left(U_{j+1}\right)+R_{j+\frac{1}{2}} \Phi_{j+\frac{1}{2}}\right] .
$$

Here $R_{j+\frac{1}{2}}$ is the eigenvector matrix of the Jacobian $A$ evaluated at some symmetric average between $U_{j}$ and $U_{j+1}$.

Second-order Symmetric TVD Scheme: The elements of the $\Phi_{j+\frac{1}{2}}$ denoted by $\left(\phi_{j+\frac{1}{2}}^{l}\right)^{S}$ for a general second-order symmetric TVD scheme [5] are

$$
\left(\phi_{j+\frac{1}{2}}^{l}\right)^{S}=-\lambda\left(a_{j+\frac{1}{2}}^{l}\right)^{2} \hat{Q}_{j+\frac{1}{2}}^{l}-\psi\left(a_{j+\frac{1}{2}}^{l}\right)\left[\alpha_{j+\frac{1}{2}}^{l}-\hat{Q}_{j+\frac{1}{2}}^{l}\right] .
$$

The value $a_{j+\frac{1}{2}}^{l}$ is the characteristic speed $a$ evaluated at some symmetric average between $U_{j}$ and $U_{j+1}$. The function $\psi$ is

$$
\psi(z)= \begin{cases}|z| & |z| \geq \delta_{1} \\ \left(z^{2}+\delta_{1}^{2}\right) / 2 \delta_{1} & |z|<\delta_{1}\end{cases}
$$


Here $\psi(z)$ in equation (4.3b) is an entropy correction to $|z|$ where $\delta_{1}$ is a small positive parameter (see references $[7,27]$ for a formula for $\delta_{1}$ ). Since all of the test problems contain unsteady shocks, $\delta_{1}$ is set to zero in all of the computations. For steady-state problems containing strong shock waves, a proper control of the size of $\delta_{1}$ is very important, especially for hypersonic blunt-body flows. See reference [7] for detailed discussion and numerical examples. Examples of the limiter function $\hat{Q}_{j+\frac{1}{2}}^{l}$ can be expressed as

$$
\begin{aligned}
& \hat{Q}_{j+\frac{1}{2}}^{l}=\operatorname{minmod}\left(\alpha_{j-\frac{1}{2}}^{l}, \alpha_{j+\frac{1}{2}}^{l}\right)+\operatorname{minmod}\left(\alpha_{j+\frac{1}{2}}^{l}, \alpha_{j+\frac{3}{2}}^{l}\right)-\alpha_{j+\frac{1}{2}}^{l} \\
& \hat{Q}_{j+\frac{1}{2}}^{l}=\operatorname{minmod}\left(\alpha_{j-\frac{1}{2}}^{l}, \alpha_{j+\frac{1}{2}}^{l}, \alpha_{j+\frac{3}{2}}^{l}\right) \\
& \hat{Q}_{j+\frac{1}{2}}^{l}=\operatorname{minmod}\left[2 \alpha_{j-\frac{1}{2}}^{l}, 2 \alpha_{j+\frac{1}{2}}^{l}, 2 \alpha_{j+\frac{3}{2}}^{l}, \frac{1}{2}\left(\alpha_{j-\frac{1}{2}}^{l}+\alpha_{j+\frac{3}{2}}^{l}\right)\right] .
\end{aligned}
$$

The minmod function of a list of arguments is equal to the smallest number in absolute value if the list of arguments is of the same sign, or is equal to zero if any arguments are of opposite sign. Here $\alpha_{j+\frac{1}{2}}^{l}$ are elements of

$$
\alpha_{j+\frac{1}{2}}=R_{j+\frac{1}{2}}^{-1}\left(U_{j+1}-U_{j}\right)
$$

Second-Order Upwind TVD Scheme: The elements of the $\Phi_{j+\frac{1}{2}}$ denoted by $\left(\phi_{j+\frac{1}{2}}^{l}\right)^{U}$ for a second-order upwind TVD scheme, originally developed by Harten, and later modified and generalized by Yee $[7,28]$, are

$$
\left(\phi_{j+\frac{1}{2}}^{l}\right)^{U}=\sigma\left(a_{j+\frac{1}{2}}^{l}\right)\left(g_{j+1}^{l}+g_{j}^{l}\right)-\psi\left(a_{j+\frac{1}{2}}^{l}+\gamma_{j+\frac{1}{2}}^{l}\right) \alpha_{j+\frac{1}{2}}^{l} .
$$

The function $\sigma(z)=\frac{1}{2}\left[\psi(z)-\lambda z^{2}\right]$ and

$$
\gamma_{j+\frac{1}{2}}^{l}=\sigma\left(a_{j+\frac{1}{2}}^{l}\right)\left\{\begin{array}{ll}
\left(g_{j+1}^{l}-g_{j}^{l}\right) / \alpha_{j+\frac{1}{2}}^{l} & \alpha_{j+\frac{1}{2}}^{l} \neq 0 \\
0 & \alpha_{j+\frac{1}{2}}^{l}=0
\end{array} .\right.
$$

Examples of the limiter function $g_{j}^{l}$ can be expressed as

$$
\begin{aligned}
& g_{j}^{l}=\operatorname{minmod}\left(\alpha_{j-\frac{1}{2}}^{l}, \alpha_{j+\frac{1}{2}}^{l}\right) \\
& g_{j}^{l}=\left(\alpha_{j+\frac{1}{2}}^{l} \alpha_{j-\frac{1}{2}}^{l}+\left|\alpha_{j+\frac{1}{2}}^{l} \alpha_{j-\frac{1}{2}}^{l}\right|\right) /\left(\alpha_{j+\frac{1}{2}}^{l}+\alpha_{j-\frac{1}{2}}^{l}\right) \\
& g_{j}^{l}=\left\{\alpha_{j-\frac{1}{2}}^{l}\left[\left(\alpha_{j+\frac{1}{2}}^{l}\right)^{2}+\delta_{2}\right]+\alpha_{j+\frac{1}{2}}^{l}\left[\left(\alpha_{j-\frac{1}{2}}^{l}\right)^{2}+\delta_{2}\right]\right\} /\left[\left(\alpha_{j+\frac{1}{2}}^{l}\right)^{2}+\left(\alpha_{j-\frac{1}{2}}^{l}\right)^{2}+2 \delta_{2}\right] \\
& g_{j}^{l}=S \cdot \max \left\{0, \min \left(2\left|\alpha_{j+\frac{1}{2}}^{l}\right|, S \cdot \alpha_{j-\frac{1}{2}}^{l}\right), \min \left(\left|\alpha_{j+\frac{1}{2}}^{l}\right|, 2 S \cdot \alpha_{j-\frac{1}{2}}^{l}\right)\right\} ; S=\operatorname{sgn}\left(\alpha_{j+\frac{1}{2}}^{l}\right) .
\end{aligned}
$$

In limiter $(4.5 e) \delta_{2}$ is a small parameter (see reference $[1]$ ). 


\subsection{MUSCL Approach}

MUSCL Approach Using an Approximate Riemann Solver: The numerical flux function $\widetilde{H}_{j+\frac{1}{2}}$ for a MUSCL-type approach of an upwind scheme as described in Yee [29] using an approximate Riemann solver can be expressed as

$$
\tilde{H}_{j+\frac{1}{2}}=\frac{1}{2}\left[F\left(U_{j+\frac{1}{2}}^{R}\right)+F\left(U_{j+\frac{1}{2}}^{L}\right)-\widehat{R}_{j+\frac{1}{2}} \hat{\Phi}_{j+\frac{1}{2}}\right]
$$

where the elements of $\widehat{\Phi}_{j+\frac{1}{2}}$ are

$$
\begin{aligned}
& \hat{\phi}_{j+\frac{1}{2}}^{l}=\left|\hat{a}_{j+\frac{1}{2}}^{l}\right| \hat{\alpha}_{j+\frac{1}{2}}^{l} \\
& \hat{\alpha}_{j+\frac{1}{2}}=\hat{R}_{j+\frac{1}{2}}^{-1}\left(U_{j+\frac{1}{2}}^{R}-U_{j+\frac{1}{2}}^{L}\right) .
\end{aligned}
$$

Here $\hat{a}_{j+\frac{1}{2}}^{l}$ are the eigenvalues and $\widehat{R}_{j+\frac{1}{2}}$ is the eigenvector matrix of $A$, evaluated using an approximate Riemann solver between the two states $U_{j+\frac{1}{2}}^{R}$ and $U_{j+\frac{1}{2}}^{L}$; that is,

$$
\begin{aligned}
& \hat{a}_{j+\frac{1}{2}}^{l}=a^{l}\left(U_{j+\frac{1}{2}}^{R}, U_{j+\frac{1}{2}}^{L}\right) \\
& \widehat{R}_{j+\frac{1}{2}}=R\left(U_{j+\frac{1}{2}}^{R}, U_{j+\frac{1}{2}}^{L}\right) .
\end{aligned}
$$

The variables $U_{j+\frac{1}{2}}^{R}$ and $U_{j+\frac{1}{2}}^{L}$ are obtained by interpolating neighboring state variables, using limiters. However, there are options in choosing the types of dependent variables in applying limiters for system cases. Namely, for the Euler equations, one can impose limiters on the conservative, primitive, or characteristic variables. For the numerical tests only one option has been used, imposing the limiters on the set of variables $W=(\rho, u, \rho \epsilon)$. Then, if we let $P$ and $P^{-1}$ denote transformation operators such that $U=P W$ and $W=P^{-1} U$, the vectors $U_{j+\frac{1}{2}}^{R}$ and $U_{j+\frac{1}{2}}^{L}$ for a second-order in time, second-order in space MUSCL approach can be defined as

$$
\begin{aligned}
& U_{j+\frac{1}{2}}^{L}=P\left(W_{j}^{n+\frac{1}{2}}+\frac{1}{2} \tilde{g}_{j}\right) \\
& U_{j+\frac{1}{2}}^{R}=P\left(W_{j+1}^{n+\frac{1}{2}}-\frac{1}{2} \tilde{g}_{j+1}\right) .
\end{aligned}
$$

Here $\tilde{g}_{j}$ is defined as in equations (4.5c)-(4.5f), except the arguments will be $\left(W_{j+1}^{n}-W_{j}^{n}\right)$ and $\left(W_{j}^{n}-W_{j-1}^{n}\right)$, with $W_{j}^{n+\frac{1}{2}}=P^{-1} U_{j}^{n+\frac{1}{2}}$, where

$$
U_{j}^{n+\frac{1}{2}}=U_{j}^{n}-\frac{\lambda}{2}\left[F\left(P\left(W_{j}^{n}+\frac{1}{2} \tilde{g}_{j}\right)\right)-F\left(P\left(W_{j}^{n}-\frac{1}{2} \tilde{g}_{j}\right)\right)\right]
$$


MUSCL Approach Using Flux-Vector Splittings: The numerical flux $\quad \tilde{H}_{j+\frac{1}{2}}$ for either flux-vector splitting can be expressed as

$$
\tilde{H}_{j+\frac{1}{2}}=F^{+}\left(U_{j+\frac{1}{2}}^{L}\right)+F^{-}\left(U_{j+\frac{1}{2}}^{R}\right)
$$

where $F^{ \pm}\left(U_{j+\frac{1}{2}}^{L, R}\right)$ are evaluated using either the generalized Steger-Warming flux-vector splitting or the generalized van Leer flux-vector splitting. The vectors $U_{j+\frac{1}{2}}^{R}$ and $U_{j+\frac{1}{2}}^{L}$ are the same as in equations (4.7).

\section{Test Cases}

Six test cases will be presented for various combinations of Mach numbers, densities and pressures. They are all one-dimensional Riemann problems. The left- and right-hand-side states of the initial conditions are tabulated in table 1 . The cases have been ordered in the direction of increasing maximum Mach numbers encountered in the flow.

Table 1. Initial conditions for the test cases.

\begin{tabular}{|c|l|l|r|r|r|r|r|}
\hline Case & State & $\begin{array}{l}\text { Density, } \\
\mathrm{kg} / \mathrm{m}^{3}\end{array}$ & $\begin{array}{c}\text { Pressure, } \\
\mathrm{N} / \mathrm{m}^{2}\end{array}$ & $\begin{array}{c}\text { Temp. } \\
K\end{array}$ & $\begin{array}{c}\text { Energy, } \\
(\mathrm{m} / \mathrm{sec})^{2}\end{array}$ & $\begin{array}{c}\text { Velocity, } \\
\mathrm{m} / \mathrm{sec}\end{array}$ & Mach No. \\
\hline Case A & Left & 0.0660 & $9.8410^{4}$ & 4390 & $7.2210^{6}$ & 0 & 0.0 \\
& Right & 0.0300 & $1.5010^{4}$ & 1378 & $1.4410^{6}$ & 0 & 0.0 \\
Case B & Left & 1.4000 & $9.8810^{5}$ & 2438 & $2.2210^{6}$ & 0 & 0.0 \\
& Right & 0.1400 & $9.9310^{3}$ & 2452 & $2.2410^{6}$ & 0 & 0.0 \\
Case C & Left & 1.2900 & $1.0010^{5}$ & 272 & $1.9510^{5}$ & 0 & 0.0 \\
& Right & 0.0129 & $1.0010^{4}$ & 2627 & $2.7510^{6}$ & 0 & 0.0 \\
Case D & Left & 1.0000 & $6.5010^{5}$ & 2242 & $2.0010^{6}$ & 0 & 0.0 \\
& Right & 0.0100 & $1.0010^{3}$ & 346 & $2.5010^{5}$ & 0 & 0.0 \\
Case E & Left & 0.0100 & $5.7310^{2}$ & 199 & $1.4410^{5}$ & 2200 & 7.8 \\
& Right & 0.1400 & $2.2310^{4}$ & 546 & $4.0010^{5}$ & 0 & 0.0 \\
& Left & 0.0100 & $5.7310^{2}$ & 199 & $1.4410^{5}$ & 4100 & 14.6 \\
& Right & 0.0100 & $5.7310^{2}$ & 199 & $1.4410^{5}$ & -4000 & -14.5 \\
\hline
\end{tabular}

The thermodynamic properties of equilibrium air are obtained from the curve fits of Srinivasan et al. [26]. These curve fits give analytic expressions for $\bar{\gamma}$ in several ranges of density and internal energy. The values of $\gamma$ are then calculated from the derivatives of these analytical expressions. The numerical solution is compared with an "exact solution" computed by solving the Rankine-Hugoniot jump conditions and integrating numerically the characteristic equations in the expansion fan. Figure 1 shows the distribution of Mach number and of the two quantities $\gamma$ and $\bar{\gamma}$ defined in equation (3.8). Not only the changes in their values but also the differences between them are an indication of departure from the perfect gas case. These differences do not necessarily occur at very high temperatures, but at intermediate temperatures when the vibration is excited and when the dissociation reactions start.

The six cases have also been tested for a perfect gas $(\gamma=1.4)$, with the same initial conditions of density and internal energy. Indeed, though they correspond to significant variations of the 
the thermodynamic properties of the gas, the conditions of test cases $A, B$, and $D$ are not very severe. On the other hand, test case $C$ corresponds to a large jump of internal energy through the contact discontinuity, and cases $\mathrm{E}$ and $\mathrm{F}$ present strong shocks at different Mach numbers. Since real-gas effects occur in general in flows with large discontinuities, it is important to identify this last effect in order to estimate the performances of the methods for the real gases.

\section{Numerical Results}

Five different second-order explicit schemes have been tested for the cases described in table 1, namely,

a. The symmetric TVD scheme (4.3), non-MUSCL

b. The upwind TVD scheme (4.5), non-MUSCL

c. The upwind TVD scheme (4.6), MUSCL

d. The generalized van Leer flux-vector splitting (4.8), MUSCL

e. The generalized Steger-Warming flux-vector splitting (4.8), MUSCL

Schemes (a) and (b) follow the non-MUSCL approach, schemes (c)-(e) follow the MUSCL approach. For the test cases presented here, the same approximate Riemann solver is used in schemes (a)-(c). The distribution of six variables $\gamma, \bar{\gamma}, u / c, \epsilon, u$, and $\rho$ are shown together because the differences between the schemes do not always occur for the same variable. After a presentation of the results obtained with these five schemes, some issues concerning the comparison of the differencing methods and of the Riemann solvers are addressed.

\subsection{Numerical Experiments}

The numerical experiments were done with a uniform grid. The number of discretization points is 141 in cases A-D (table 1), and only 81 for cases $E$ and F because in the latter cases the expansion fan is replaced by a shock. The origin $x=0$ corresponds to the initial discontinuity between the left and right states. In the grid, this origin corresponds to the middle of the interval number 70 for cases A-D, 16 for case $E$, and 35 for case $F$. The time for stopping the computation has been chosen for each case in order to use the full computational domain. In the case of equilibrium air, for a $\Delta x$ normalized to $0.1 \mathrm{~m}$, these stopping times, are $t=3.5$ $m s e c$ for case A, $t=3.0 \mathrm{msec}$ for case $\mathrm{B}, t=5.0 \mathrm{msec}$ for case $\mathrm{C}, t=3.2 \mathrm{msec}$ for case $\mathrm{D}$, $t=7.0 \mathrm{msec}$ for case $\mathrm{E}$ and $t=6.0 \mathrm{msec}$ for case $\mathrm{F}$. These times have not been changed for the perfect gas except that $t=4.0 \mathrm{msec}$ for case $\mathrm{F}$ because the differences in $\gamma$ dramatically change the shock motion.

The whole set of results presented has been obtained by using the same limiter function for each scheme. Scheme (a) uses the limiter (4.3e). Schemes (b)-(e) use the limiter (4.5e). For the numerical values used in all the test cases presented, a value of $10^{-7}$ for an appropriately normalized parameter $\delta_{2}$ was found to be adequate. Results using scheme (b) with the limiter (4.5d) on the nonlinear fields and limiter (4.5e) on the linear field can be found in references $[5,7]$.

The results presented for the approximate Riemann solver have been obtained with two different averages. The results for cases $A, D$, and $F$ used the generalized Roe average (section 
$3.1)$, with one modification. The projection was done on the straight line defined by equation (3.12), and the scale factor was chosen as $\bar{h}$. Thus equations (3.17a) and (3.17b) were replaced by

$$
\frac{\bar{\chi}}{\bar{h}}=\left\{\frac{1}{2}\left[\frac{\left(\chi^{L}+\chi^{R}\right)}{\bar{h}} \Delta \tilde{\epsilon}-\left(\kappa^{L}+\kappa^{R}\right) \bar{h} \Delta \rho\right] \Delta \tilde{\epsilon}+\Delta p \bar{h} \Delta \rho\right\} /\left(\Delta \tilde{\epsilon}^{2}+(\bar{h} \Delta \rho)^{2}\right)
$$

and

$$
\bar{\kappa}=\left\{\Delta p \Delta \tilde{\epsilon}-\frac{1}{2}\left[\frac{\left(\chi^{L}+\chi^{R}\right)}{\bar{h}} \Delta \tilde{\epsilon}-\left(\kappa^{L}+\kappa^{R}\right) \bar{h} \Delta \rho\right] \bar{h} \Delta \rho\right\} /\left(\Delta \tilde{\epsilon}^{2}+(\bar{h} \Delta \rho)^{2}\right)
$$

The results for the cases $\mathrm{B}, \mathrm{C}$, and $\mathrm{E}$ used modifications of the average of Carofano $[21]$ :

$$
\begin{aligned}
\frac{\bar{c}^{2}}{\bar{h}} & =0.5\left(\frac{\left(c^{L}\right)^{2}}{h^{L}}+\frac{\left(c^{R}\right)^{2}}{h^{R}}\right) \\
\bar{\kappa} & =0.5\left(\kappa^{L}+\kappa^{R}\right) \\
\bar{\chi} & =\bar{c}^{2}-\bar{\kappa} \bar{h} .
\end{aligned}
$$

This average recovers Roe's average for a perfect gas but does not satisfy Roe's property "U" for a real gas.

The time-step limit is expressed in term of a CFL number that has been defined as the maximum value of $(|u|+c) \Delta t / \Delta x$. For those cases in which the maximum value occurs in a subsonic region, such as in test case $C$, it was found that the flux-vector splitting requires a more stringent CFL limit. When $|u / c|$ is less than one, the value of $(|u|+c) \Delta t / \Delta x$ for the generalized van Leer flux-vector splitting had to be multiplied by the factor [17]

$$
k_{1}=(|u|+c)(\gamma+3) /[2 \gamma+|u / c|(3-\gamma)]
$$

whereas for the Steger-Warming flux-vector splitting a less stringent modification was found adequate, using a factor

$$
k_{2}=1 /(0.8+0.2|u / c|) \text {. }
$$

This CFL number has been set equal to 0.9 except for case $C$. In case $C$ it has been fixed at 0.5 for the upwind non-MUSCL and MUSCL schemes, and at 0.9 for the symmetric scheme. We briefly describe here the results obtained for each case.

Test case A: The variations of $\gamma$ and $\bar{\gamma}$ are important. The plots for test case A (figs. 2,3) show that for the intermediate states of the numerical solution at the contact discontinuity, the variations of $\gamma$ are not even monotonic. Actually, in the Mollier diagram, $\gamma$ is not a monotonic function of energy, and in that particular case, the two end states of the contact discontinuity are located on either side a minimum for $\gamma$. Even though the distribution of such quantities 
as density or energy is monotonic, an oscillation necessarily occurs for $\gamma$. This behavior may be the cause of a small wiggle for the velocity distribution at the contact discontinuity, which does not appear for the perfect gas. Otherwise, the comparison between the perfect gas and the real gas indicates no deficiency in the generalization techniques. The differences between the schemes are very small for this problem. They all capture the shock within three points. The best results are obtained with the schemes (b), (c) and (d). No significant difference appears between the symmetric scheme (a) and the upwind schemes, either non-MUSCL (b) or MUSCL (c).

Test case B: The intensity of the shock in case B is greater than in case A (figs. 4, 5). The capture of the shock is slightly sharper with the schemes (a)-(c) using the approximate Riemann solver. In this case, no particular problem can be detected at the contact discontinuity. The symmetric scheme (a), however, captures it within more points than the upwind schemes. Looking in particular at the velocity distribution, the most regular solution is given by the scheme (c) in this case. Again, the behavior of all the numerical solutions are quite similar using either the perfect gas or the real gas.

Test case $C$ : Case $\mathrm{C}$ is a difficult test for all schemes, either for the real (fig. 6) gas or perfect gas (fig. 7). There are noticeable errors on the levels of the velocities and on the position of the shock. The errors are a little greater for the real gas. All the schemes have problems at the contact discontinuity for the real gas. Especially for scheme (e), using the generalized Steger-Warming flux splitting, the value 0.9 of the CFL number without the correction yields wiggles that propagate everywhere on the solution. For the perfect gas, only schemes (d) and (e) using the flux-vector splitting technique have a problem. This may be related to the fact that unlike for the five others cases for the perfect gas, the absolute value of the Mach number crosses 1.0 at the contact discontinuity. At the shock also, the schemes have some problems. The resolution is very sensitive to the time-step, especially for scheme (b). This was the reason for choosing the CFL value of 0.5 for the upwind schemes and 0.9 for the symmetric scheme, which seems to give the best results at the shock. As the same problem occurs both for the real gas and the perfect gas, this case shows the limits of the numerical methods even more than those of their extensions to a real gas.

Test case D: Case D (fig. 8,9 ) is characterized by a large transonic rarefaction wave. The discontinuity, which occurs in the middle for most of the variables on the real gas case, is also present on the distribution of $\gamma$ and $\bar{\gamma}$ for the exact solution. The tables from reference [26] fit the state equation with continuous functions on a set of regions in density and energy which covers the physical range of interest. However, continuity is only loosely ensured at the boundaries of these regions. The discontinuity observed here corresponds to a density boundary. Here, the discontinuities in the values given by the curve fits for the pressure and its derivatives is approximately $5 \%$. In that case, only scheme (e) using the generalized StegerWarming flux-vector splitting recovers a smooth solution at that point. As in case $\mathrm{C}$, but with less intensity, an error on the levels of energy and velocity appears, either for the real gas (fig. 8) or the perfect gas (fig. 9). This error is less important with the non-MUSCL schemes (a) and (b). The results are shown for a value of the CFL number equal to 0.9, which corresponds to the sharpest resolution of the shock. At a lower value of the CFL number (0.5), the energy overshoot at the contact discontinuity is almost completly released for the nonMUSCL schemes (a) and (b). On the contrary, this does not help with the MUSCL schemes 
(c)-(e).

Test case $E$ : In case $\mathrm{E}$ (fig. 10,11), the strong shock on the left and the contact discontinuity correspond to large jumps on the thermodynamic variables. The shock is almost stationary and is captured within the same number of points with all the schemes. However, the lowest oscillation just behind the shock is achieved by scheme (d) using the generalized van Leer flux-vector splitting. The contact discontinuity is best captured with the symmetric scheme (a). All the results present some wiggles on the energy and the velocity in the region between the shocks. However, the levels are straighter with schemes (a) and (e). For schemes (c)-(e) the choice of the limiter function is more critical. They blow up when using limiter (4.5d) or (4.5f). Again, the behavior of the schemes is very similar for the real gas and the perfect gas. However, for the perfect gas, the wiggles are slightly smaller.

Test case F: The situation for case $F$ (figs. 12, 13) is almost the same as for case $\mathrm{E}$. The approximate Riemann solver captures the shock within one point less than the flux-vector splittings. The extra point in the shock given by flux-vector splittings (d) and (e), yields a value of $\gamma$ that does not lie between the limits on each side. As in case $A$, this is due to the nature of the state equation. The bump observed in the distribution of energy and density is at the location of the contact discontinuity. It is mainly a result of errors introduced at the very first time-steps, when the whole phenomenon is represented, without enough accuracy, on a few space intervals (tests on the same case at lower Mach number show that the error reduces with the value of the Mach number). Some schemes seem to damp this error more effectively, for example, scheme (e) using the Steger-Warming flux-vector splitting and symmetric TVD scheme (a). Moreover, the solutions obtained with symmetric TVD scheme (a) and upwind scheme (b) non-MUSCL schemes are very similar since this particular example contains shock waves only. Because of the slight asymmetry introduced in the velocities of the initial states, the velocity of the intermediate state of the Riemann problem is not exactly zero. However, the strenghts of the two shocks are the same and their numerical solution should be symmetrical. As can be seen from the density plots, this is not exactly true for schemes (d) and (e) using the flux-vector splittings. Moreover, using schemes (d) and (e), a steady solution is formed within a few time-steps, with the two initial states separated at one point by a state that has a nearly zero velocity. This seems to be due to the fact that the states $U_{j+\frac{1}{2}}^{R}$ and $U_{j-\frac{1}{2}}^{L}$ defined by equations (4.7) around this point remain supersonic. In order to overcome this difficulty, it has been necessary to impose a zero value on the slope of the velocity at the intermediate point during the first time-steps. Otherwise, the effects of limiters and of the state equation are similar to case $\mathbf{E}$.

\subsection{Comparison of the Differencing Techniques}

Parts (a)-(c) of figures 2-13 provide comparisons of the symmetric TVD scheme, the upwind TVD scheme, and the MUSCL scheme with the same approximate Riemann solver for cases A through F (table 1). On the basis of the numerical tests, some general observations can be made about the numerical resolution and the computational efficiency of the methods. Since it was not possible to display the results obtained with all the limiter functions $((4.3 \mathrm{c})-(4.3 \mathrm{e})$; (4.5c)-(4.5f)) introduced for the different schemes, some additional comments are given about their use on the test cases. 
Numerical Resolution: The results obtained with the differencing techniques rely on the validity of the Riemann solver that they use. From this point of view the real-gas case is not theoretically different from the perfect gas. Then the conclusions found here for the accuracy are mostly related to the flow conditions in Mach number and intensity of the discontinuities. For relatively gentle cases like $\mathrm{A}$ and $\mathrm{B}$, the techniques are hardly distinguishable. Greater differences appear in the representation of the shock between the non-MUSCL and the MUSCL approach in case $\mathrm{C}$ and also in cases $\mathrm{E}$ and $\mathrm{F}$. Particularly in case $\mathrm{C}$, the non-MUSCL approach appears to be slightly more sensitive to the time-step. For the treatment of the contact discontinuities the non-MUSCL approach seems to be slightly better, especially in case D. But the major differences are between the symmetric scheme and the two upwind schemes. Although the upwind schemes give better solutions for the rapidly unsteady cases like A-D, especially for contact discontinuities, the situation is reversed in cases $\mathrm{E}$ and $\mathrm{F}$ where the motion of the shock is slow. The levels are more flat with the symmetric scheme; moreover, the capture of the contact discontinuity is sharper with the symmetric scheme in case E. Finally, cases $\mathrm{C}$ and $D$ seem to be at the accuracy limit of the methods, within the number of points used here for the discretization of the problems.

Influence of the Limiter Functions: The limiter function is an important parameter for the TVD schemes. The choices used here correspond to what we considered as a good compromise between stability and accuracy on the whole set of test cases. In particular for the cases A, B and $\mathrm{D}$, the treatment of contact discontinuities and expansion waves can be improved by using limiters (4.5d) or (4.5f) instead of (4.5e) for the upwind schemes (b)-(e). However, when using limiter (4.5d) or (4.5f) in cases $E$ and $F$, some instabilities have been found with the MUSCL schemes near the shocks for high Mach numbers. Similarly, limiter (4.5f) with either upwind scheme yields an overshoot at the contact discontinuity for case $\mathrm{C}$. The different tests seem to show that the influence of the limiter is the same as for a perfect gas. Detailed discussions of this subject can be found in $[5,7]$ for the non-MUSCL schemes, and in $[1,9]$ for the MUSCL scheme.

Computational Effort: the use of a curve fit for the computation of thermodynamic variables like the pressure and sound speed from the conservative variables can be considerably more time consuming than the simple analytic formulas existing for a perfect gas. The non-MUSCL approach evaluates these properties once at the center of each cell. On the other hand, the need for computing the formulas of the Riemann solvers at each interface requires two such evaluations per cell and per space dimension for the MUSCL approach. For the one-dimensional tests, this difference was not very critical. For multidimensional calculation, the differences can be larger and depend greatly on the efficiency of the software package used for the curve fits.

\subsection{Comparison of the Riemann Solvers}

Parts (c)-(d) of figures 2-13 compare the three Riemann solvers for the MUSCL scheme. In this part, we are mostly interested in checking the validity of the Riemann solvers. For this purpose, we analyze here the effects of the two different phenomena occurring in these flows, the large discontinuities and the real gas state equation.

Influence of the Mach Number and of Large Discontinuities: Since these methods have been 
used for a long time with a perfect gas, remarks will be made only about the treatment of large discontinuities which are found especially in cases $C, E$ and F. All three methods seem to be able to handle shocks corresponding to fairly high Mach numbers. However, the wiggles that appear in the subsonic region in cases $E$ and $F$ tend to increase with the Mach number, for the perfect and real gases. Form this point of view, the flux-vector splitting methods which are more dissipative show slightly fewer of these wiggles. On the other hand, case $\mathrm{C}$ seems to show some limits for these Riemann solvers in transient flows with large discontinuities. In general, the approximate Riemann solver is more accurate for the contact discontinuities. Especially in case $C$, it gives a better resolution where flux-vector splitting methods have trouble at the contact discontinuity, even with the perfect gas.

Real Gas Effects: In the test conditions used, the comparisons of the different Riemann solvers is not changed very much by the use of equilibrium air. The advantages of the generalized van Leer flux-vector splitting over the generalized Steger-Warming flux-vector splitting remain the same as for the perfect gas in general. The three generalization techniques involve a free parameter. Though the choices used in the numerical tests correspond to natural extensions of the perfect-gas techniques, a wide variety of possibilities has not been explored. The way of determining the best choice for the most accurate and stable results is not clear. In the case of the approximate Riemann solver, even the necessity of using an average satisfying Roe's property " $U$ " is an open question. The average (6.1) did not yield a noticeable improvement on the results obtained with the other one (6.2). Another important fact is that flux-vector splittings make use of the sound speed only, whereas approximate Riemann solvers of the Roe-type make use of the thermodynamic derivatives $\chi$ and $\kappa$ of equation (4.2). These thermodynamic derivatives put more stringent requirements on the curve fit that represents the thermodynamic properties of the gas. In this regard, the curve fits of Srinivasan et al. may be deficient for the approximate Riemann solver as can be seen from figure 5, case $D$. One probably needs more improved curve fits than those of reference [26] before a definite conclusion can be drawn on the accuracy of the different Riemann solvers and schemes. From this point of view, the generalized Steger-Warming flux-vector splitting, which is more diffusive than the two others Riemann solvers, is also less sensitive to a lack of regularity in the curve fits.

\section{Concluding Remarks}

For the purpose of calculations in gas dynamics with an equilibrium real gas, the numerical tests show that the simple extensions to a real gas for the flux-vector splitting or the approximate Riemann solver presented in this paper are valid. The main effect of using a real gas equation of state is to exacerbate the problems of the methods for large discontinuities. Test case $\mathrm{C}$ is an example of such a situation. Similarly, it seems difficult to give a scaling of the methods. Depending on the case, each one presents some drawbacks or some advantages.

Looking at the Riemann solvers in general, the advantages of the generalized van Leer fluxvector splitting over the generalized Steger and Warming formulation remain for a real gas, with slightly less dissipation at the discontinuities. The approximate Riemann solver gives results very similar to the generalized van Leer flux-vector splitting formulation. Looking at the numerical schemes, the main difference seems to occur between the upwind and the 
symmetric TVD schemes. The upwind schemes give better results in strongly transient flows. On the other hand the symmetric scheme has a better stability in regions of low Mach number and produces shock resolution similar to that of the Steger-Warming flux-vector splitting in the MUSCL approach.

The difference in computational effort required by the three Riemann solvers is small. The main difference lies in the MUSCL and non-MUSCL approaches. For one-dimensional problems, the operations-count between the non-MUSCL and MUSCL approaches is within $30 \%$ for a perfect gas. However, due to extra evaluation in the curve fitting between the left and right states in an equilibrium real gas for the MUSCL formulation, additional computation is required for the MUSCL approach. The effective amount of additional computation increases nonlinearly as the spatial dimension increases. None of the differences observed for the explicit versions seems to be decisive for the one-dimensional tests, but factors such as stability and computational efficiency need further investigation in multidimensional tests, which are in progress. Preliminary study shows certain advantages of the approximate Riemann solver over the flux-vector splitting approaches. The present results also indicate that the state equation does not have a very large effect on the general behavior of these methods for a wide range of flow conditions. The ease of extending these methods to multidimensional problems and/or to efficient implicit algorithms will be a more important issue for application to hypersonic flow calculations.

\section{References}

[1] B. van Leer, Towards the Ultimate Conservation Difference Scheme V, A Second-Order Sequel to Godunov's Method, J. Comp. Phys., 32, 101-136 (1979).

[2] P. Colella and P.R. Woodward, The Piecewise Parabolic Method (PPM) for Gas Dynamical Simulations, J. Comp. Phys. 54, 174-201 (1984).

[3] A. Harten, On a Class of High Resolution Total-Variation-Stable Finite-Difference Schemes, SIAM J. Num. Anal, 21, 1-23 (1984).

[4] P.L. Roe, Some Contributions to the Modelling of Discontinuous Flows, Lectures in Applied Mathematics, 22, 163-194 (Amer. Math. Soc., Providence, R.I., 1985).

[5] H.C. Yee, Construction of Explicit and Implicit Symmetric TVD Schemes and Their Applications, J. Comput. Phys., 68, 151-179 (1987); also NASA TM-86775, July 1985.

[6] H.C. Yee, Numerical Experiments with a Symmetric High-Resolution Shock-Capturing Scheme, Proc. 10th Int. Conf. on Numerical Methods in Fluid Dynamics, June 1986, Beijing, China; also NASA TM-88325, June 1986.

[7] H.C. Yee, Upwind and Symmetric Shock-Capturing Schemes, NASA TM-89464, May 1987; also to appear, proceedings of the "Seminar on Computational Aerodynamics," Dept. Mech. Engin., University of Calif., Davis, Spring Quarter, 1986.

[8] Y.J. Moon and H.C. Yee, Numerical Simulation by TVD Schemes of Complex Shock Reflections from Airfoils at High Angle of Attack, AIAA Paper 87-0350, Jan. 1987.

[9] W.K. Anderson, J.L. Thomas and B. van Leer, A Comparison of Finite Volume FluxVector Splittings for Euler Equations, AIAA Paper No. 85-0122, 1985. 
[10] M. Borrel and J.-L. Montagné, Numerical Study of a Non-centered Scheme with Application to Aerodynamics, AIAA Paper No. 85-1497, 1985.

[11] S. K. Godunov, A. V. Zabrodin and G. P. Prokopov, A Computational Scheme for TwoDimensional Non-Stationary Problems of Gas Dynamics and Calculation of the Flow from a Shock Wave Approaching a Stationary State, USSR Comp. Math. Phys., 1, 1187-1219 (1961).

[12] P.L. Roe, Approximate Riemann Solvers, Parameter Vectors, and Difference Schemes, J. Comp. Phys., 43, 357-372 (1981).

[13] L.C. Huang, Pseudo-Unsteady Difference Schemes for Discontinuous Solutions of SteadyState, One-Dimensional Fluid Dynamics Problems, J. Comp. Phys., 42, 195-211 (1981).

[14] A. Harten, P. D. Lax and B. van Leer, On upstream Difference and Godunov-Type Schemes for Hyperbolic Conservative Laws, SIAM Review, 25, 35-61 (1983).

[15] S. Osher and F. Solomon, Upwinded Schemes for Hyperbolic Systems of Conservation Laws, Math. Comp., 38, 339-377 (1981).

[16] J.L. Steger and R.F. Warming, Flux-Vector Splitting of the Inviscid Gasdynamic Equations with Application to Finite Difference Methods, J. Comput. Phys., 40, 263-293 (1981).

[17] B. van Leer, Flux-Vector Splitting for the Euler Equations, ICASE Report 82-30; Sept., 1982.

[18] R. H. Sanders and K. H. Prendergast, The Possible Relation of 3-Kiloparsec Arm to Explosions in the Galactic Nucleus, Astrophysical Journal, 188, 489-500 (1974).

[19] P. Colella and H.R. Glaz, Efficient Solution Algorithm for the Riemann Problem for Real Gases, J. Comp. Phys., 59, 254-289 (1985).

[20] P.A. Gnoffo, R.S. McCandless and H.C. Yee, Enhancements to Program LAURA for Efficient Computation of Three-Dimensional Hypersonic Flow, AIAA Paper 87-0280, Jan. 1987.

[21] G.C. Carofano, Blast Computation Using Harten's Total Variation Diminishing Scheme, Technical Report ARLCB-TR-84029.

[22] M. Vinokur, Generalized Roe Averaging for Real Gas, NASA Contractor Report, in preparation.

[23] J.-L. Montagné , Use of an Upwinded Scheme for Simulating NonViscous Flows of Real Gas at Equilibrium, La recherche Aerospatiale, Dec. 1986.

[24] J.-L. Montagné , Rapport de Synthese finale, ONERA, 23/1285 AY, Dec. 1985.

[25] M. Vinokur and J.-L. Montagné, Generalized Flux-Vector Splitting for an Equilibrium Gas, NASA contractor report, in preparation.

[26] S. Srinivasan, J.C. Tannehill, K.J. Weilmunster, Simplified Curve Fit for the Thermodynamic Properties of Equilibrium Air, ISU-ERI-Ames 86401; ERI Project 1626; CFD15.

[27] A. Harten and J.M. Hyman, A Self-Adjusting Grid for the Computation of Weak Solutions of Hyperbolic Conservation Laws, J. Comp. Phys., 50, 235-269 (1983).

[28] H.C. Yee, Linearized Form of Implicit TVD Schemes for Multidimensional Euler and Navier-Stokes Equations, Computers and Mathematics with Applications, 12A, 413-432 (1986). 
[29] H.C. Yee, On the Implementation of a Class of Upwind Schemes for System of Hyperbolic Conservation Laws, NASA TM-86839, Sept. 1985. 


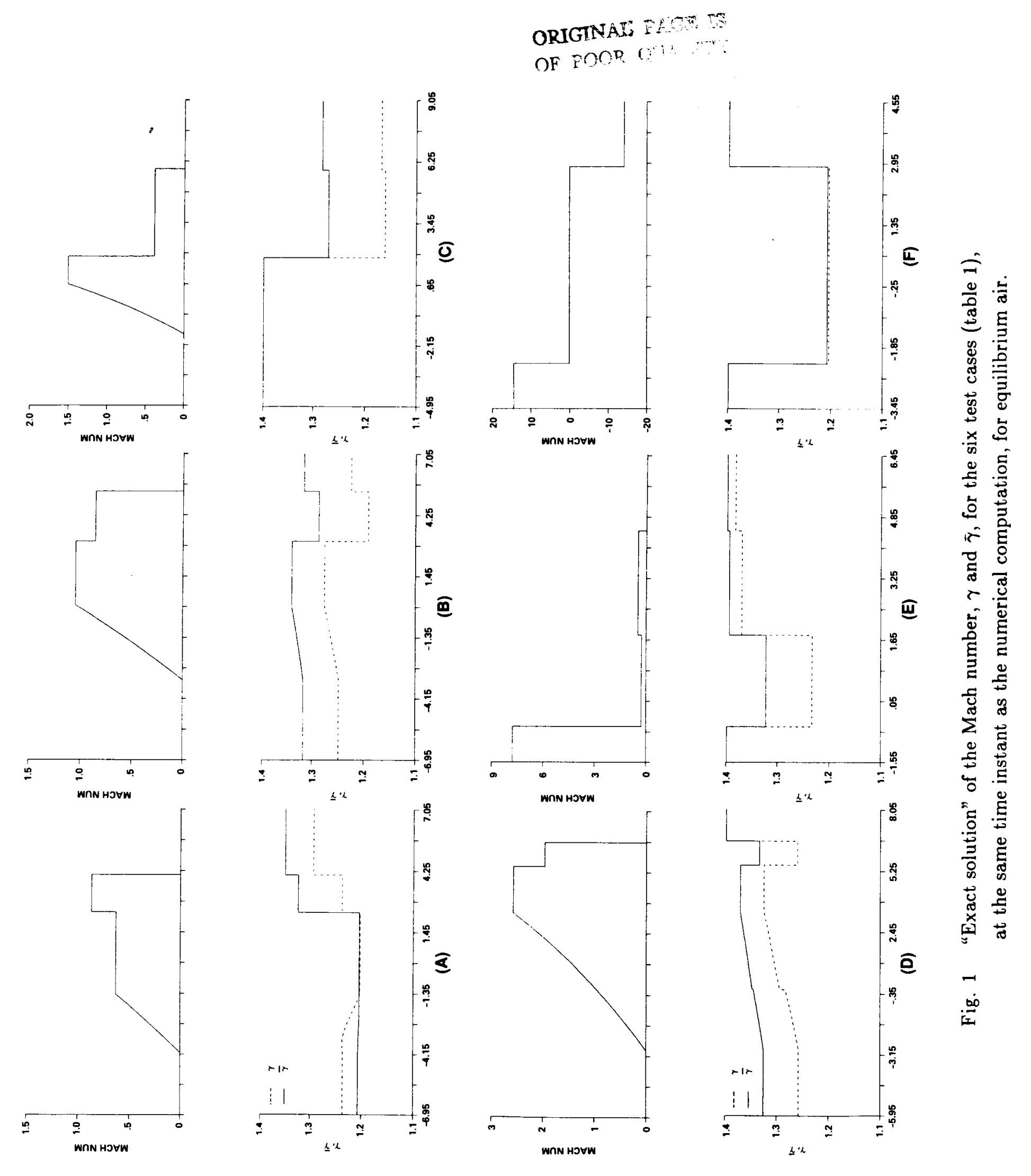




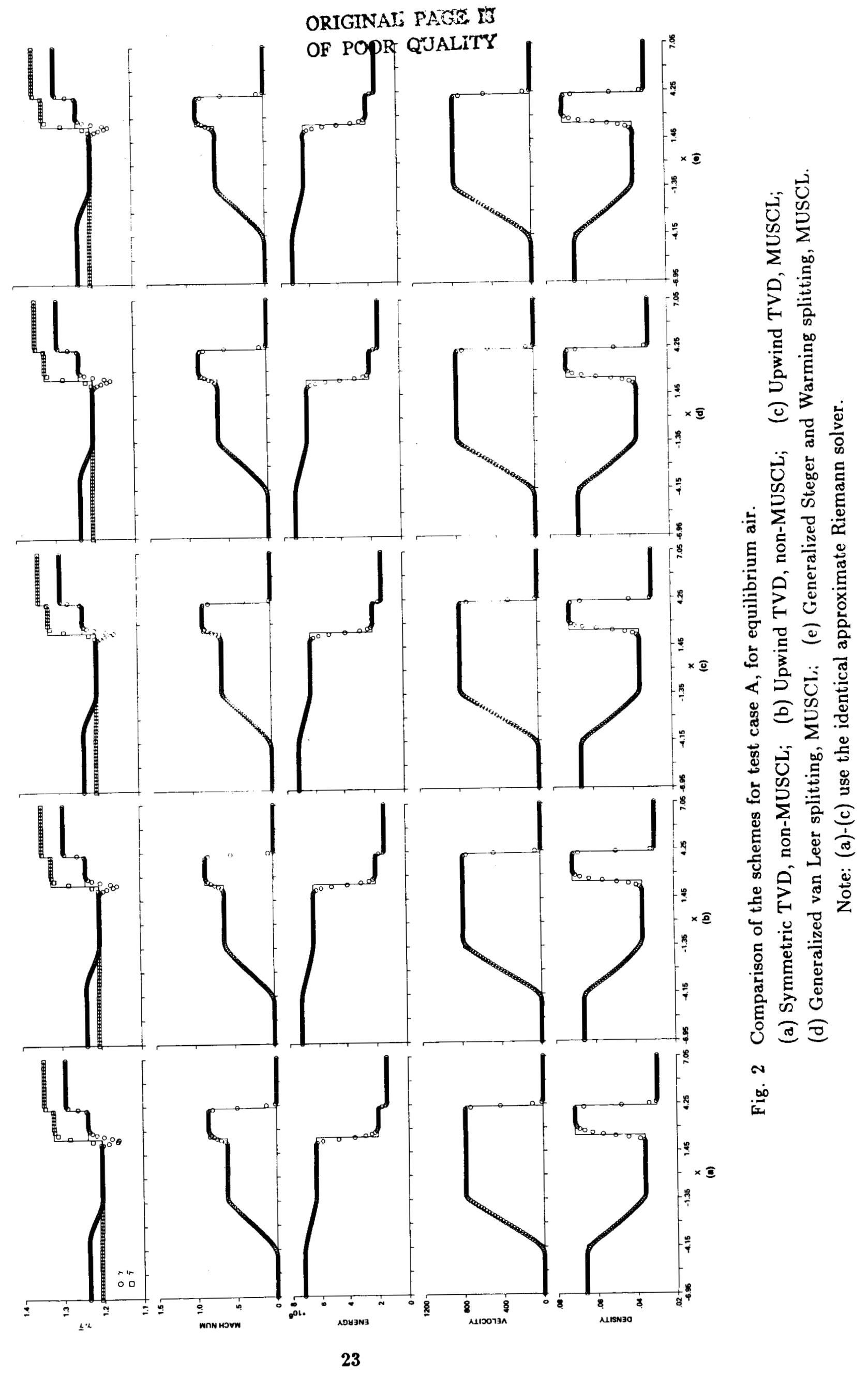




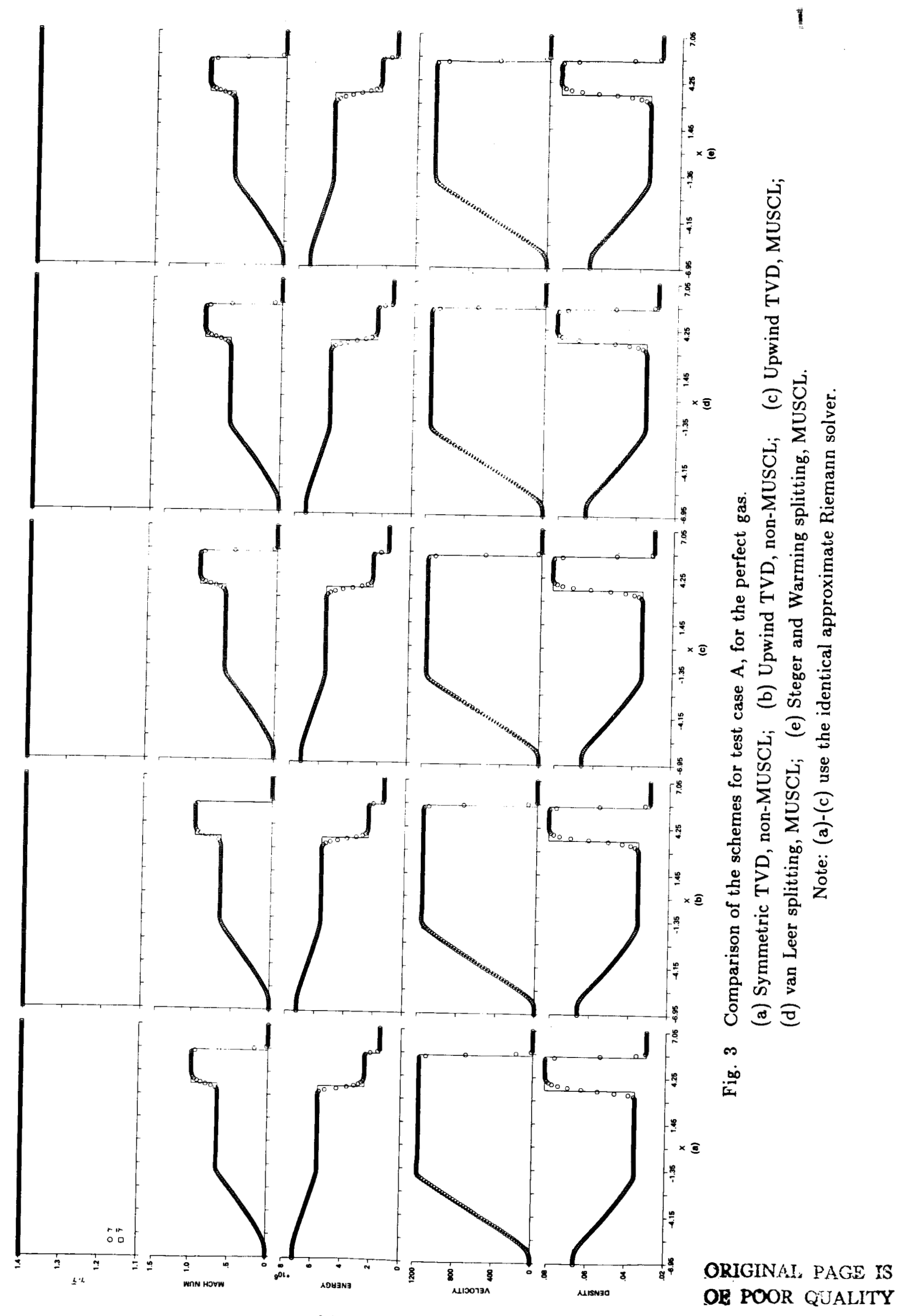




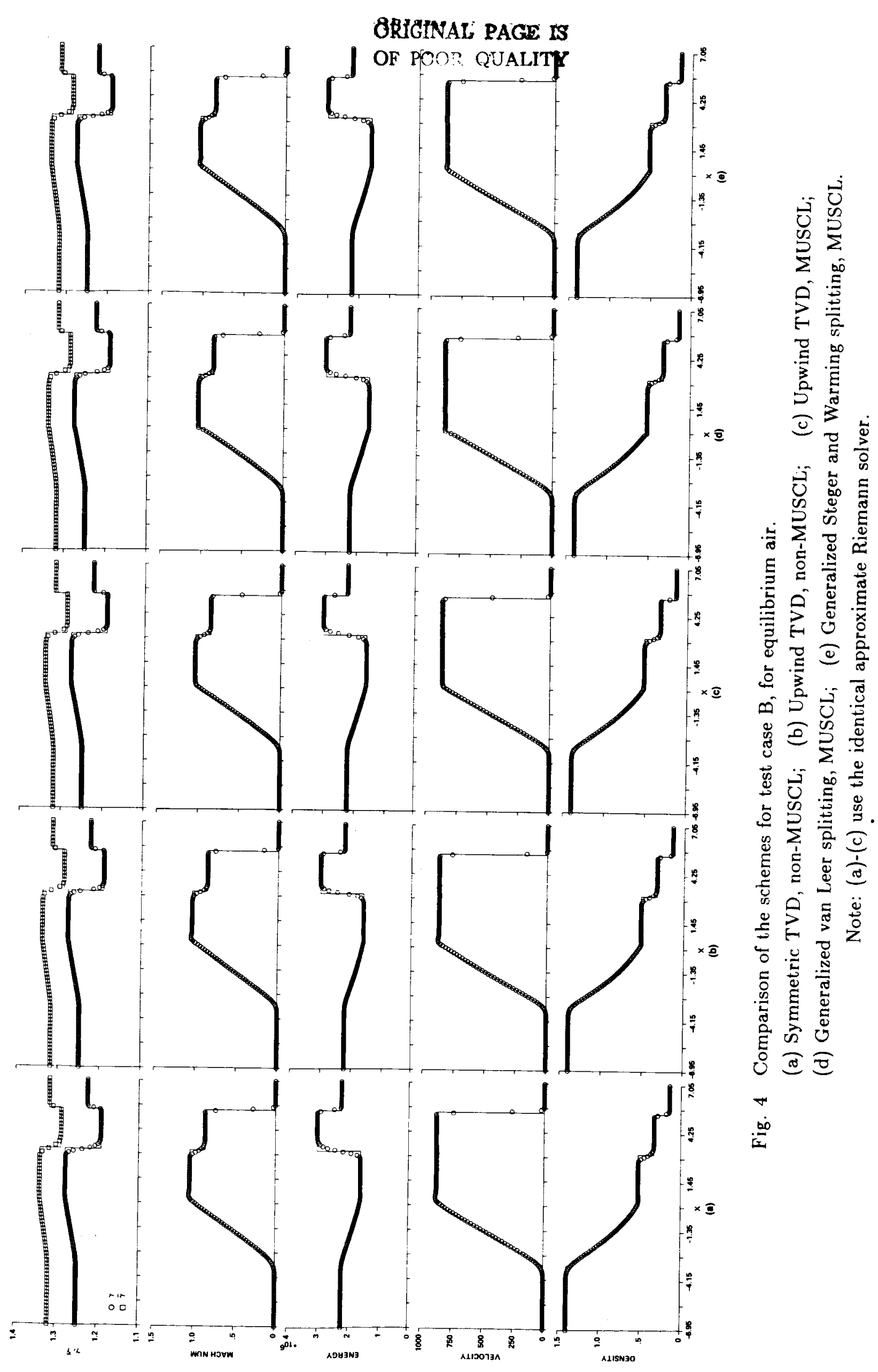




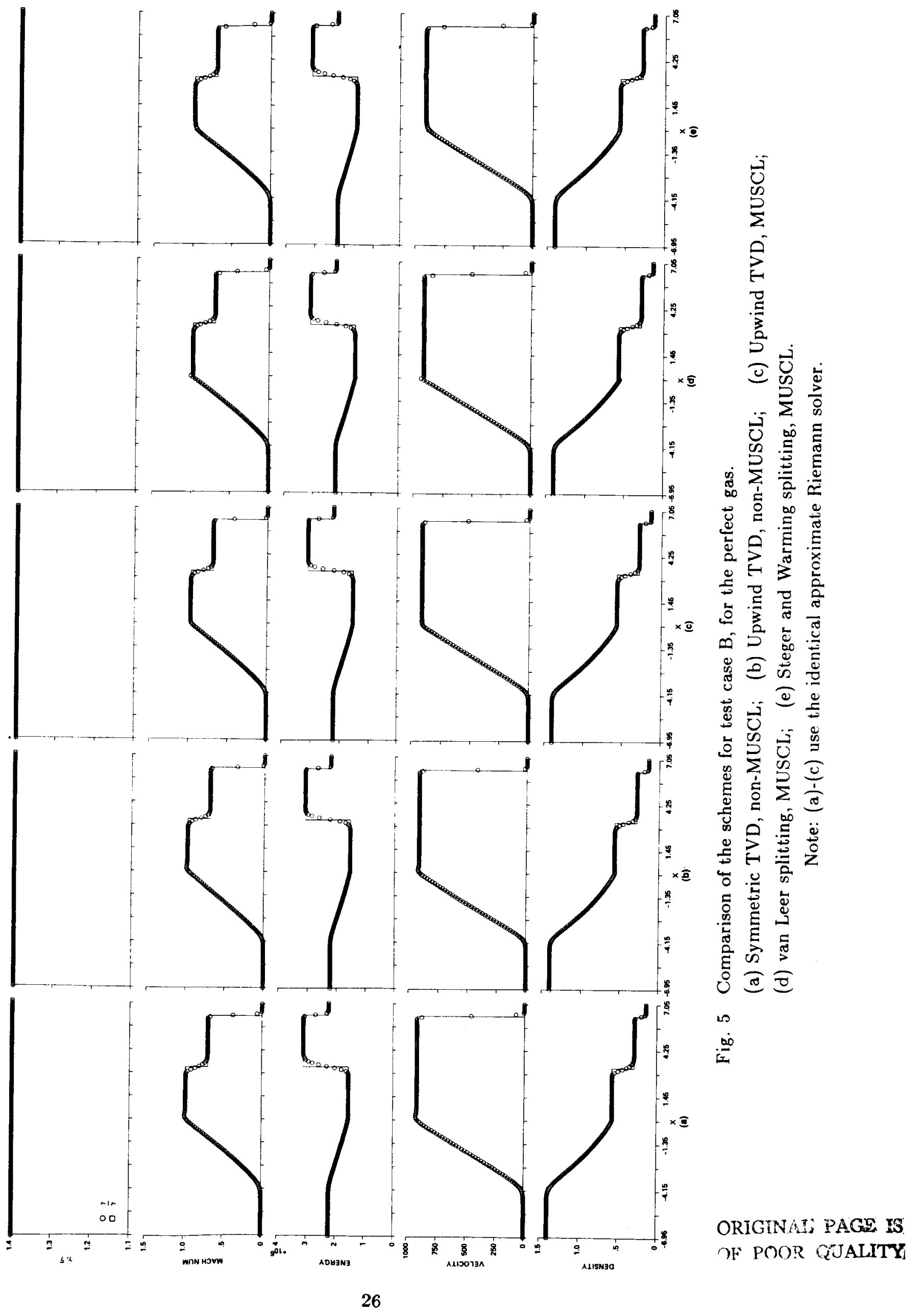




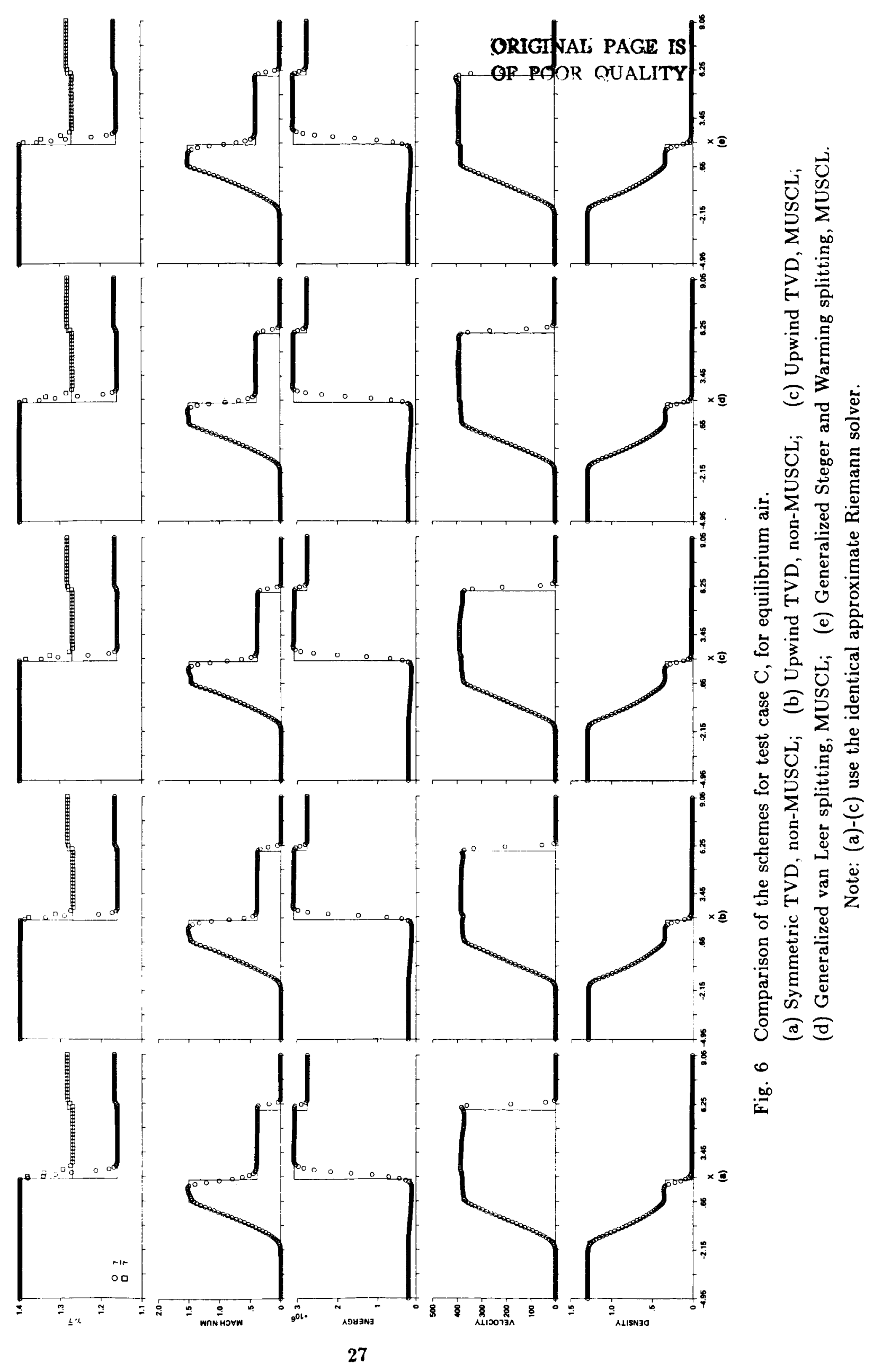




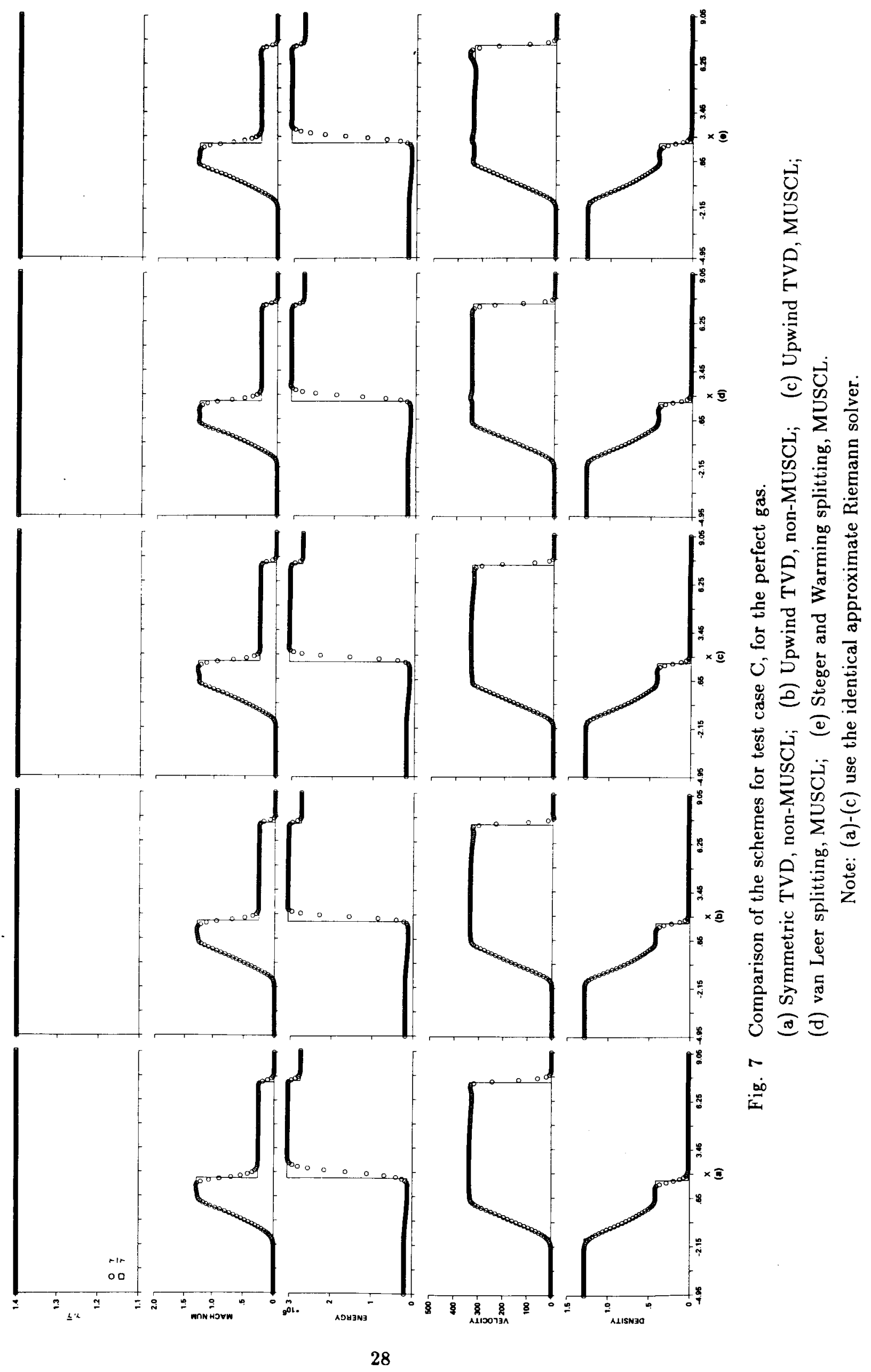




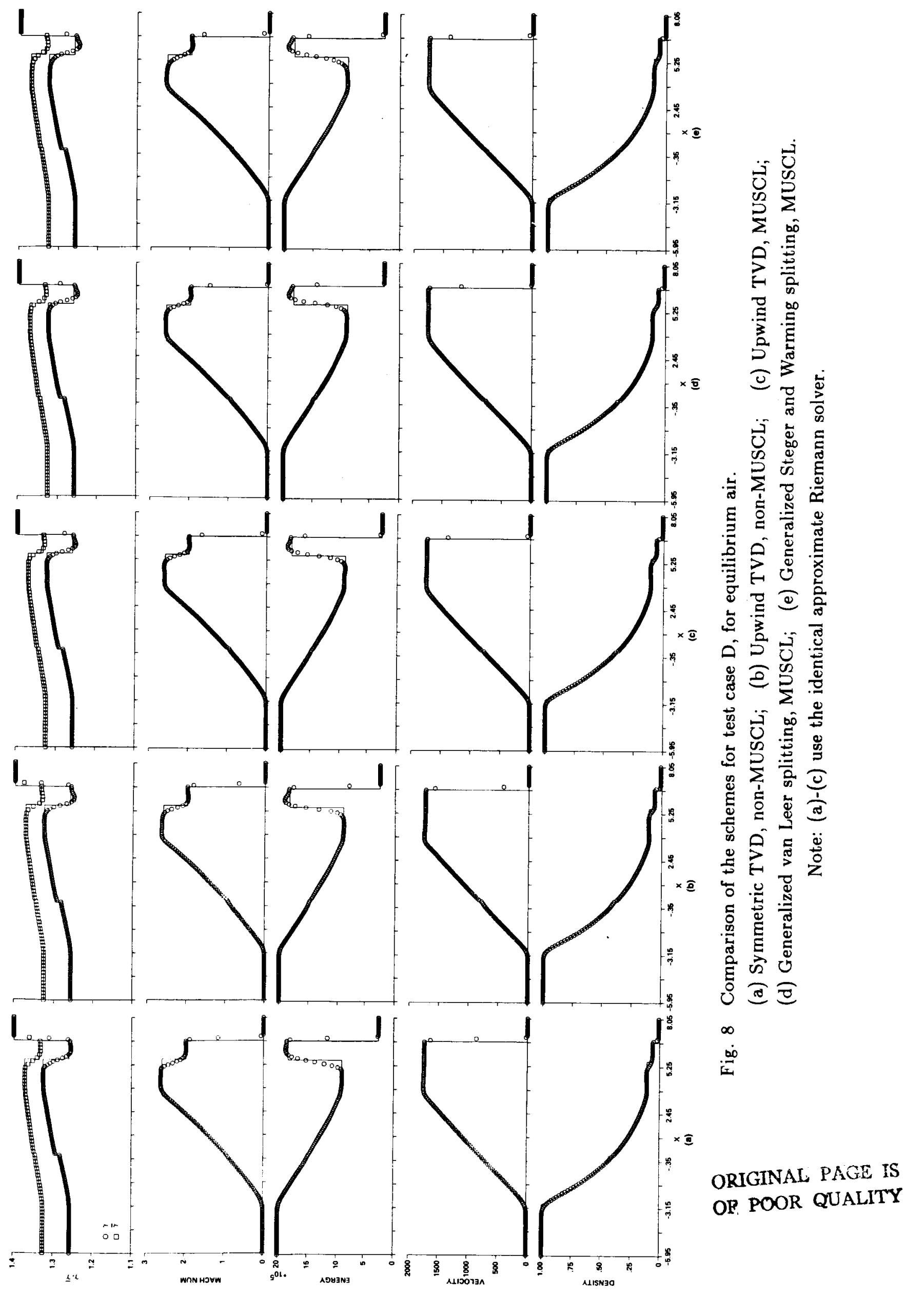




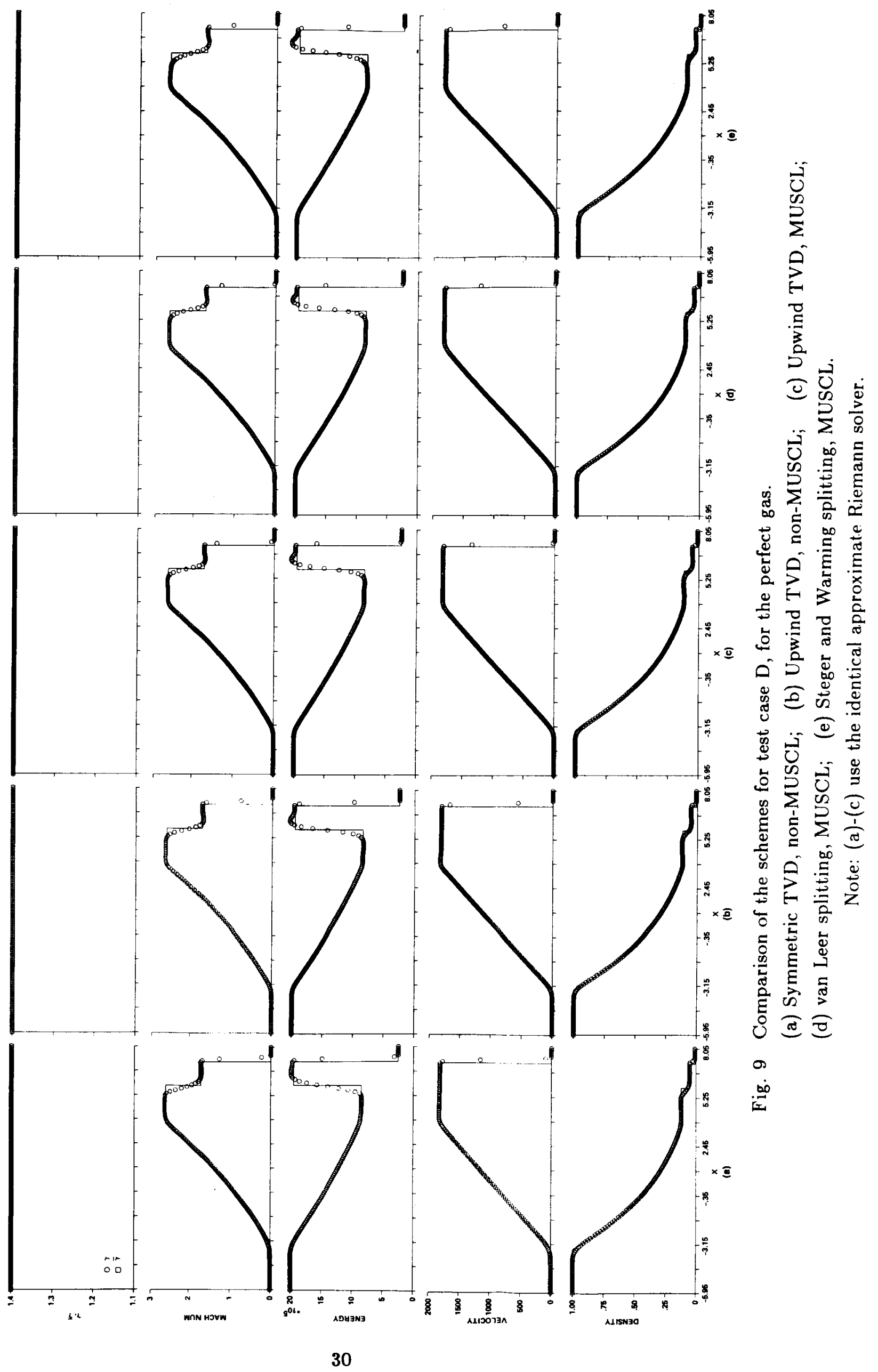




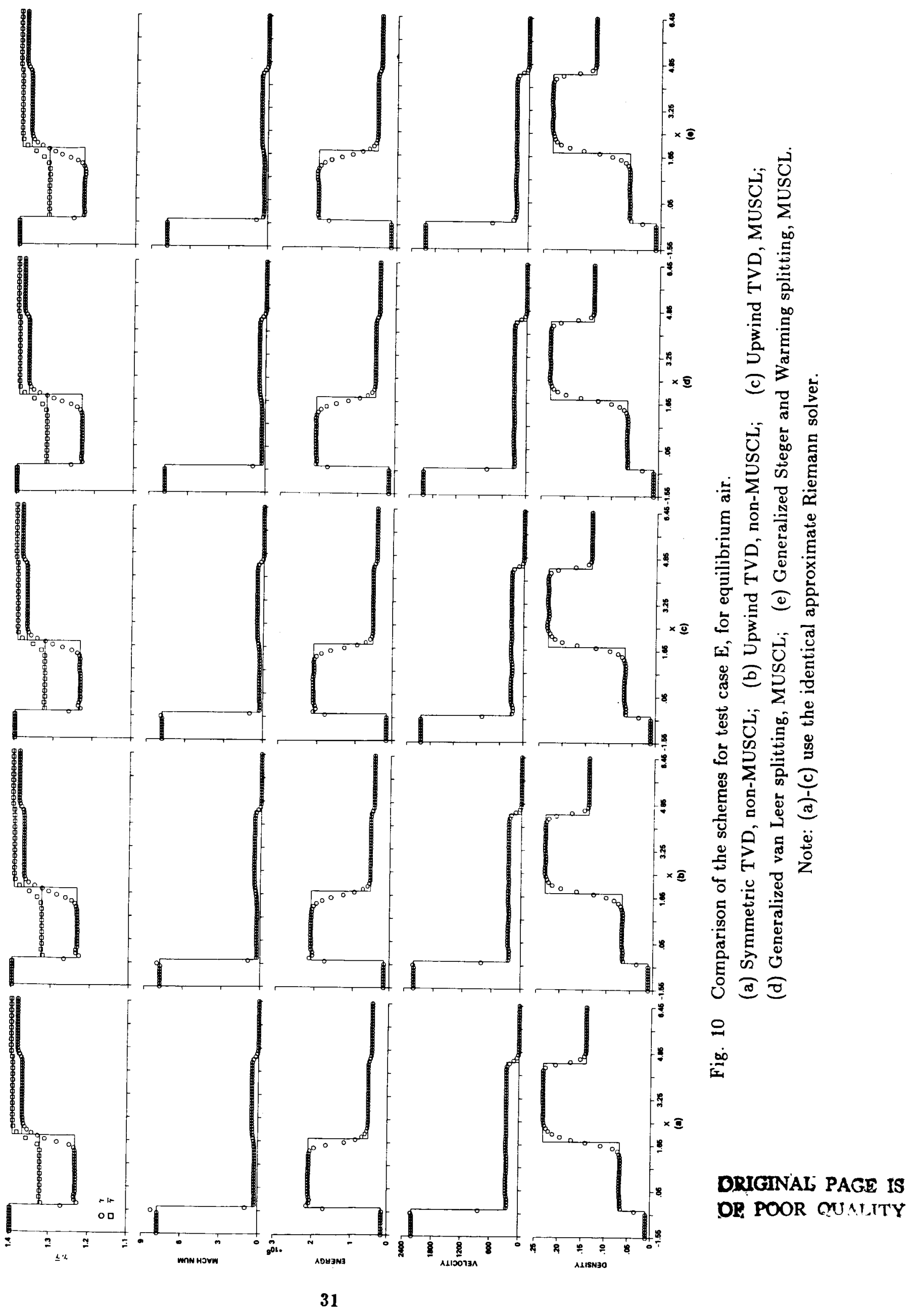




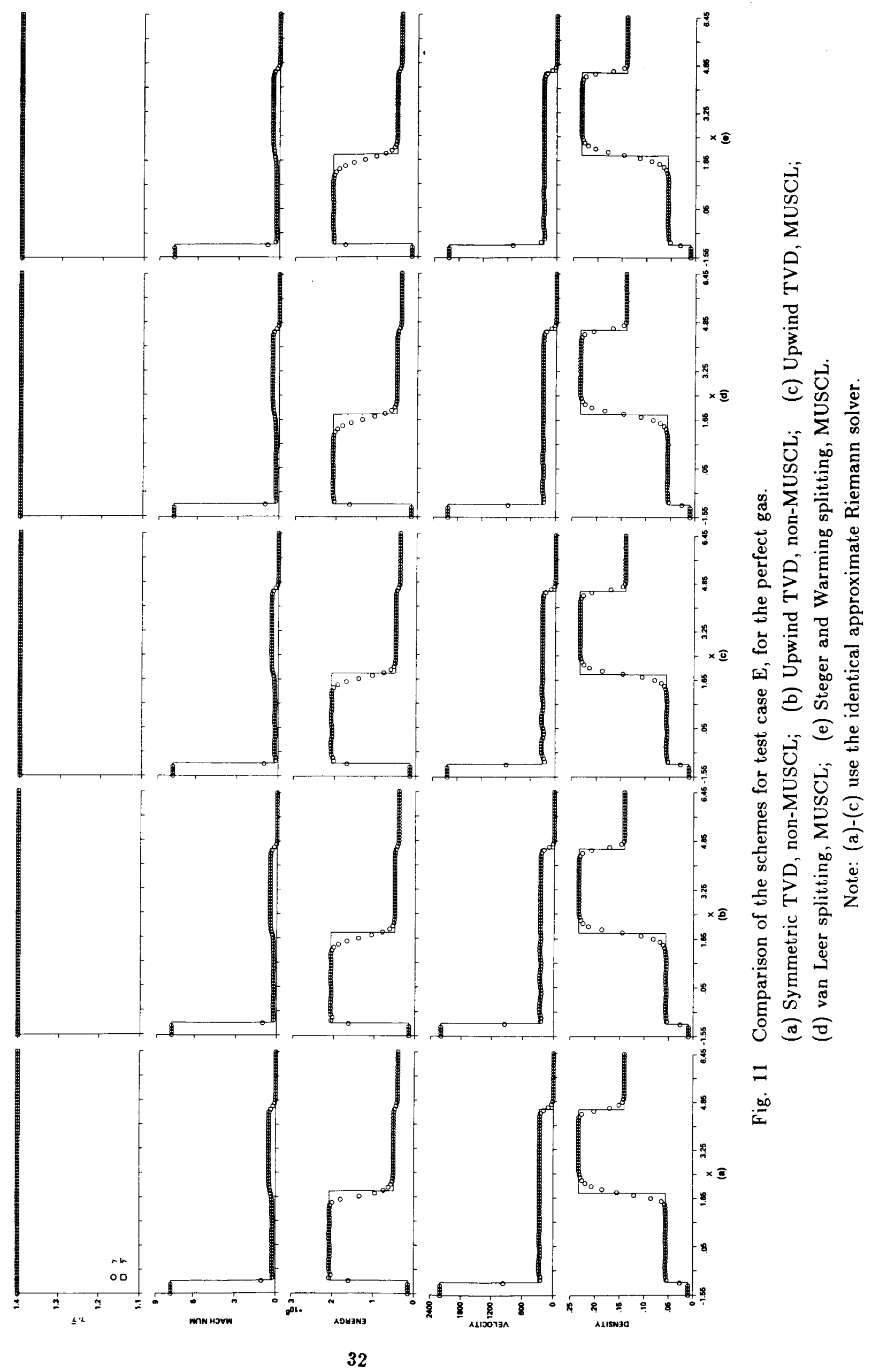




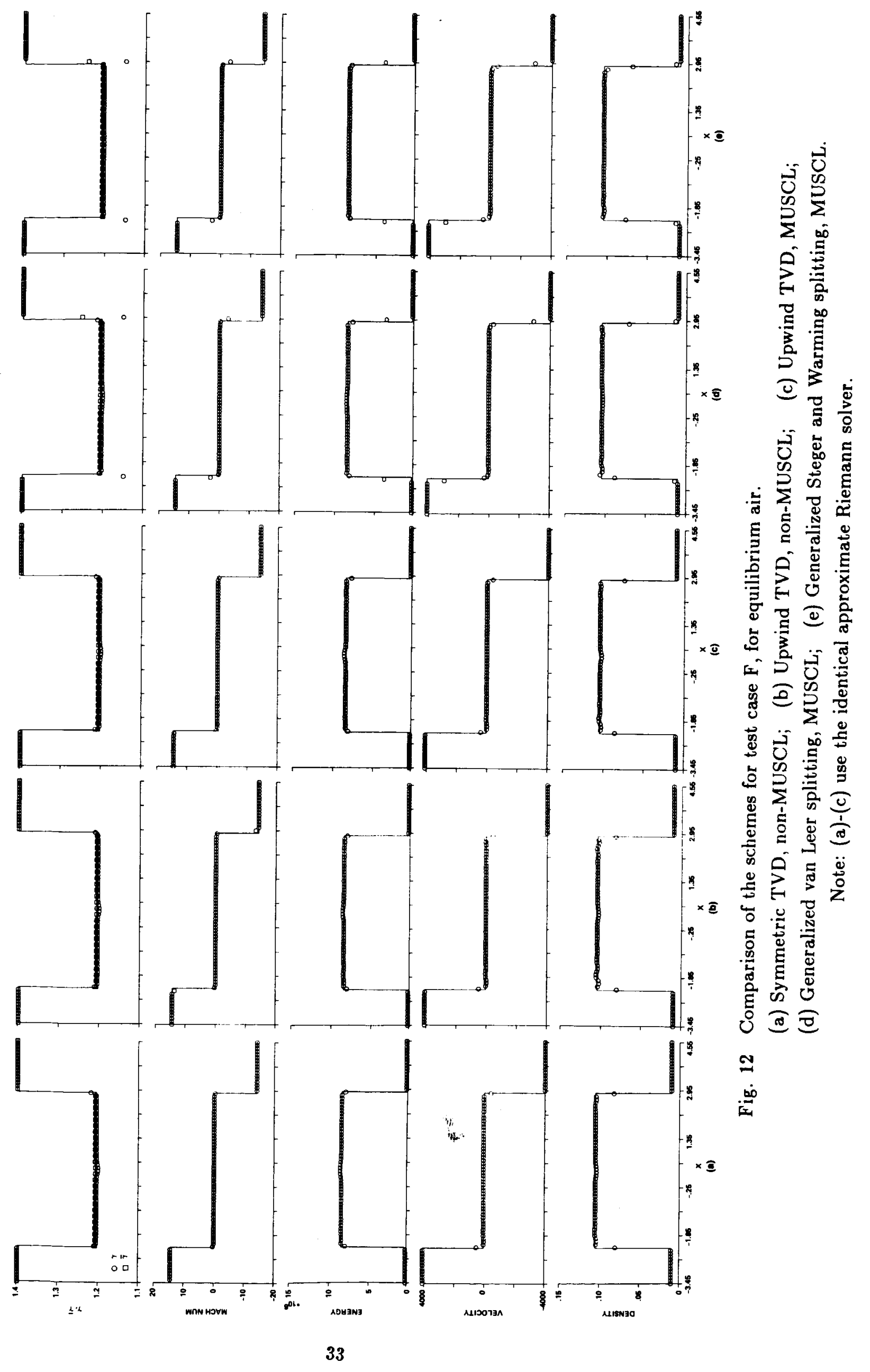




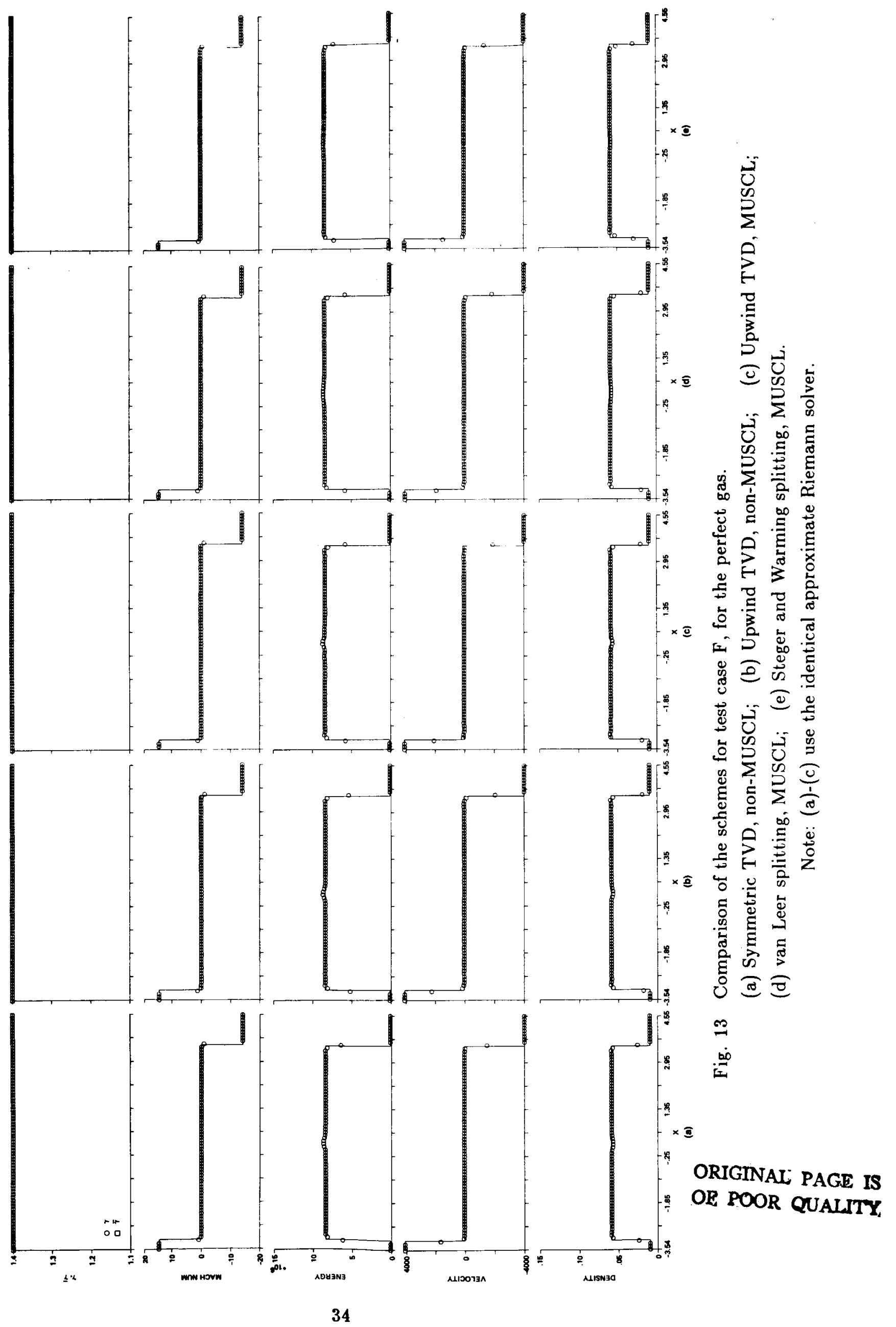




\begin{tabular}{|c|c|c|c|}
\hline \multicolumn{4}{|c|}{ Report Documentation Page } \\
\hline \multicolumn{3}{|l|}{$\begin{array}{l}\text { 1. Report No. } \\
\text { NASA TM-100004 }\end{array}$} & \\
\hline \multirow{2}{*}{\multicolumn{2}{|c|}{$\begin{array}{l}\text { 4. Title and Subtitle } \\
\text { Comparative Study of High-Resolution } \\
\text { Shock-Capturing Schemes for a Real Gas }\end{array}$}} & \multicolumn{2}{|c|}{$\begin{array}{l}\text { 5. Report Date } \\
\text { July } 1987\end{array}$} \\
\hline & & \multicolumn{2}{|c|}{ 6. Performing Organization Code } \\
\hline \multirow{2}{*}{\multicolumn{2}{|c|}{$\begin{array}{l}\text { 7. Author(s) } \\
\text { J.-L. Montagne, H. C. Yee, and M. Vinokur }\end{array}$}} & \multicolumn{2}{|c|}{$\begin{array}{l}\text { 8. Performing Organization Report No. } \\
\text { A-87274 }\end{array}$} \\
\hline & & \multicolumn{2}{|l|}{ 10. Work Unit No. } \\
\hline \multicolumn{4}{|l|}{ 9. Performing Organization Name and Address } \\
\hline \multirow{2}{*}{\multicolumn{2}{|c|}{$\begin{array}{l}\text { Ames Research Center } \\
\text { Moffett Fie1d, CA } 94035\end{array}$}} & \multicolumn{2}{|c|}{ 11. Contract or Grant No. } \\
\hline \multirow{2}{*}{\multicolumn{2}{|c|}{$\begin{array}{l}\text { 12. Sponsoring Agency Name and Address } \\
\text { Nationa1 Aeronautics and Space Administration } \\
\text { Washington, D.C. } 20546\end{array}$}} & \multicolumn{2}{|c|}{$\begin{array}{l}\text { 13. Type of Report and Period Covered } \\
\text { Technical Memorandum }\end{array}$} \\
\hline & & \multicolumn{2}{|c|}{$\begin{array}{l}\text { 14. Sponsoring Agency Code } \\
505-60\end{array}$} \\
\hline \multicolumn{4}{|c|}{ 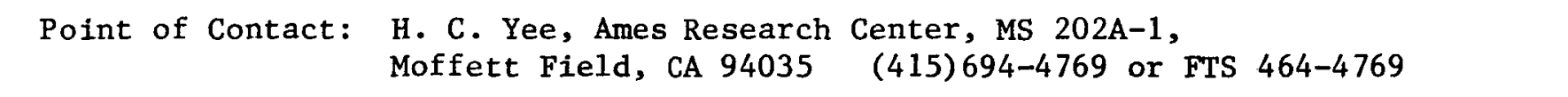 } \\
\hline \multicolumn{4}{|c|}{$\begin{array}{l}\text { 16. Abstract The recently developed second-order explicit shock-capturing.methods of the } \\
\text { van Leer, Harten and Yee types in conjunction with the generalized flux-vector } \\
\text { splittings of Vinokur and Montagne and a generalized Roe's approximate Riemann } \\
\text { solver of Vinokur for a real gas are studied. The comparison is made on different } \\
\text { one-dimensional Riemann (shock-tube) problems for equilibrium air with various } \\
\text { ranges of Mach numbers and densities and pressures. Six different Riemann prob- } \\
\text { lems are considered. These tests provide a check on the validity of the general- } \\
\text { ized formulas, since theoretical prediction of their properties appears to be } \\
\text { difficult because of the nonanalytic form of the state equation. The numerical } \\
\text { results in the supersonic and low hypersonic regimes indicate that these approaches } \\
\text { produce good shock-capturing capability and the shock resolution is not affected } \\
\text { much by the state equation of equilibrium air. The difference in shock resolution } \\
\text { between the various methods varies slightly from one Riemann problem to another } \\
\text { but the overall accuracy is very similar. The relative efficiency in terms of } \\
\text { operation count for the different methods is within 30\%. The main difference } \\
\text { between the methods lies in their versatility in extending to multidimensional } \\
\text { problems and/or implicit methods with efficient solution procedures. }\end{array}$} \\
\hline $\begin{array}{l}\text { 17. Key Words (Suggested by Author(s)) Numerical method, } \\
\text { Finite difference method, Computational } \\
\text { fluid dynamics, System of hyperbolic } \\
\text { conservation laws, Real gas, Equilibrium } \\
\text { flow, Shock capturing, Conservative } \\
\text { differencing, TVD schemes }\end{array}$ & \multicolumn{3}{|c|}{$\begin{array}{l}\text { 18. Distribution Statement } \\
\text { Unclassif ied - Unlimited } \\
\text { Subject Category - } 64\end{array}$} \\
\hline \begin{tabular}{|l|r|}
$\begin{array}{l}\text { 19. Security Classif. (of this report) } \\
\text { Unclassified }\end{array}$ & 20. Security Classif. lof t \\
Unclassified
\end{tabular} & his page & $\begin{array}{l}\text { 21. No. of pages } \\
36\end{array}$ & $\begin{array}{r}\text { 22. Price } \\
\text { A02 }\end{array}$ \\
\hline
\end{tabular}

